\title{
A RecET-assisted CRISPR-Cas9 genome editing in Corynebacterium glutamicum
}

\author{
Bo Wang ${ }^{1,2+}$, Qitiao Hu ${ }^{1,2+}$, Yu Zhang ${ }^{1,2}$, Ruilin Shi ${ }^{3}$, Xin Chai ${ }^{3}$, Zhe Liu ${ }^{1,2}$, Xiuling Shang ${ }^{1}$, Yun Zhang ${ }^{1 *}$ \\ and Tingyi Wen ${ }^{1,3,4^{*}}$ D
}

\begin{abstract}
Background: Extensive modification of genome is an efficient manner to regulate the metabolic network for producing target metabolites or non-native products using Corynebacterium glutamicum as a cell factory. Genome editing approaches by means of homologous recombination and counter-selection markers are laborious and time consuming due to multiple round manipulations and low editing efficiencies. The current two-plasmid-based CRISPR-Cas9 editing methods generate false positives due to the potential instability of Cas9 on the plasmid, and require a high transformation efficiency for co-occurrence of two plasmids transformation.

Results: Here, we developed a RecET-assisted CRISPR-Cas9 genome editing method using a chromosome-borne Cas9-RecET and a single plasmid harboring sgRNA and repair templates. The inducible expression of chromosomal RecET promoted the frequencies of homologous recombination, and increased the efficiency for gene deletion. Due to the high transformation efficiency of a single plasmid, this method enabled 10- and 20-kb region deletion, 2.5-, 5.7and 7.5-kb expression cassette insertion and precise site-specific mutation, suggesting a versatility of this method. Deletion of $\arg R$ and farR regulators as well as site-directed mutation of $\arg B$ and $p g i$ genes generated the mutant capable of accumulating L-arginine, indicating the stability of chromosome-borne Cas 9 for iterative genome editing. Using this method, the model-predicted target genes were modified to redirect metabolic flux towards 1,2-propanediol biosynthetic pathway. The final engineered strain produced $6.75 \pm 0.46 \mathrm{~g} / \mathrm{L}$ of 1,2-propanediol that is the highest titer reported in C. glutamicum. Furthermore, this method is available for Corynebacterium pekinense 1.563, suggesting its universal applicability in other Corynebacterium species.
\end{abstract}

Conclusions: The RecET-assisted CRISPR-Cas9 genome editing method will facilitate engineering of metabolic networks for the synthesis of interested bio-based products from renewable biomass using Corynebacterium species as cell factories.

Keywords: Corynebacterium glutamicum, CRISPR-Cas9, recET-encoded recombinase, Transformation efficiency, Genome editing, 1,2-Propanediol

\section{Background}

Corynebacterium glutamicum is an important platform organism for modern biotechnology to produce amino acids, organic acids, nucleic acids, and bio-based products from the cheap and renewable biomass, such as

\footnotetext{
*Correspondence: zhangyun@im.ac.cn; wenty@im.ac.cn

†Bo Wang and Qitiao Hu contributed equally to this work

${ }^{1}$ CAS Key Laboratory of Pathogenic Microbiology and Immunology, Institute of Microbiology, Chinese Academy of Sciences, Beijing 100101, China

Full list of author information is available at the end of the article
}

glucose, sucrose, and xylose [1-3]. Genome editing to modify chromosome in way of deletion, integration or replacement is an efficient manner to metabolic engineering of this bacterium for overproducing the desired products, which is preferred than the traditional random mutagenesis [4]. Systems metabolic engineering guided by rational design using the genome-scale metabolic model requires a great deal of genetic modifications in C. glutamicum genome, which are laborious and time-consuming. Therefore, it is necessary to develop an easy and efficient genome editing method with extensive

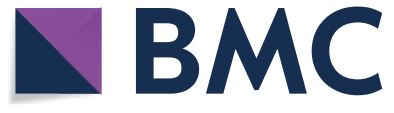

(c) The Author(s) 2018. This article is distributed under the terms of the Creative Commons Attribution 4.0 International License (http://creativecommons.org/licenses/by/4.0/), which permits unrestricted use, distribution, and reproduction in any medium, provided you give appropriate credit to the original author(s) and the source, provide a link to the Creative Commons license, and indicate if changes were made. The Creative Commons Public Domain Dedication waiver (http://creativecommons.org/ publicdomain/zero/1.0/) applies to the data made available in this article, unless otherwise stated. 
applicability to facilitate modifying the metabolic network of C. glutamicum [4-6].

The traditional approaches dependent on two rounds of homologous recombination require a non-replicating vector with a counter-selectable marker to screen target mutant in C. glutamicum ATCC 13032 [7]. The selection markers and vector elements need to be removed via a single crossover event, possibly resulting in undesired restoration of the wild type allele even in the presence of selective pressure. The counter selection by $s a c B$ gene leads to a $20-40 \%$ false-positive rate due to the spontaneous inactivation of SacB [8]. As another counter-selectable marker, upp encoding a uracil phosphoribosyltransferase is able to decrease the false-positive rate when it is concerted with I-SceI [8]. A toxin counter-selectable cassette regulated by an antitoxin switch (TCCRAS) system can achieve 100\% counter-selectable efficiencies for gene knockout and replacement [9]. To increase the marker eviction efficiency, Cre/mutant loxP system composed of cre-encoding recombinase and loxP sites are utilized to mediate reciprocal sitespecific recombination between the lox 71 and $l o x 66$ to facilitate easy removal of counter-selectable markers and undesirable vector elements $[10,11]$. In spite of high counter-selectable efficiencies, these approaches are still inefficient due to their relatively low frequencies at which homologous recombination take place. To improve homologous recombination efficiency for gene deletion, the exonuclease-recombinase RecE and RecT (RecET) from the Rac prophage of Escherichia coli are expressed on a plasmid in C. glutamicum to mediate a doublecrossover event via a dsDNA fragment containing the counter-selectable markers and homology arms $[8,12]$. With the assistance of RecET expression, a linear dsDNA is resected by RecE to expose a 3-ended single-stranded DNA (ssDNA) tail for the binding of RecT, which contributes to promote the annealing of complementary DNA strand, strand exchange and strand invasion $[8,12]$. The RecET-Cre/loxP system constructed in C. glutamicum ATCC 14067 only requires one round of recombination to obtain the target mutant [12].

The clustered regularly interspaced short palindromic repeat (CRISPR) as a bacterial immune defense system has been developed for genome editing in a wide range of organisms, such as bacteria and eukaryotes [13-17]. The type II CRISPR-Cas9 system from Streptococcus pyogenes is widely used for genome editing due to the inherent simplicity and flexibility in sequence requirements for sgRNA [13-15]. Cas9 from S. pyogenes (SpCas9) requires a single guide RNA (sgRNA) containing 20 bp complementary sequence of the target region and the SpCas9 binding scaffold to form sgRNA-SpCas9 ribonucleoprotein complex, which generates a double-strand break
(DSB) at $20 \mathrm{bp}$-designated target region after recognizing a protospacer adjacent motif (PAM) sequence NGG [13]. DSB by Cas9 cleavage in the chromosome is lethal to many bacteria deficient in the endogenous nonhomologous end joining (NHEJ) mechanism, when DSB is unable be repaired through homologous recombination (HR) [13]. Cas9-mediated killing can help select mutations introduced through HR using a donor DNA as the repair template and eliminate cells carrying the wild-type genotype [16, 17]. So far, CRISPR-Cas9 system has been developed as a powerful tool to genome editing in many bacteria [18-24].

In recent studies regarding CRISPR-Cas9 assisted genome editing of C. glutamicum, several attempts to transform the plasmid harboring the native codonencoded SpCas9 under different inducible promoters failed to obtain any colony, which might be attributed to a negative effect of its strong expression on cell viability $[25,26]$. To decrease the translation level, attempts to utilize the codon-optimized cas 9 gene and different ribosomal binding sequences succeeded to express plasmid-borne Cas9 protein [26-28]. Two plasmids-based CRISPR-Cas9 editing system consisted of inducible expression cassette of Cas9 in one plasmid and sgRNA expression cassette together with a repair template in the other plasmid $[27,28]$. However, the transformation with two plasmids harboring CRIPSR-Cas9 system yielded the abnormally large colonies in which their cas 9 genes were deleted from pCas9 plasmid, indicating the instability of pCas9. Furthermore, the manipulation procedure is relatively complicated due to the construction of two plasmids harboring genetic elements and transformation efficiency is low for the co-occurrence of the introduction of Cas9-sgRNA and repair template [double-stranded or single-stranded DNA (ssDNA)], resulting in a high requirement for transformation efficiency. The plasmidborne dsDNA template can directly repair the DSB for gene deletion via HR in NHEJ-deficient C. glutamicum; however, it is necessary to express recombinase RecT to incorporate ssDNA to repair DSB [26], indicating that HR is a key factor in CRISPR-Cas9 editing system. Compared to Cas9, Cpf1 that utilizes a T-rich PAM site has less editing targets in the GC-rich C. glutamicum genome and only reaches $5-15 \%$ of editing efficiencies for gene deletion and insertion [25]. These editing methods have disadvantage in modifying endogenous genes or integrating heterologous genes to construct synthetic pathways. Hence, it is of great importance to develop an easy and efficient CRISPR-Cas9 system to implement an extensive genome editing including gene deletion, integration and mutation in C. glutamicum for academic studies and industrial applications. 
In this study, to simplify the editing elements construction and minimize the physiological impacts of Cas9 on cells, we developed a CRISPR-Cas9 genome editing method in C. glutamicum by integrating cas 9 into chromosome and constructing a single plasmid harboring a sgRNA and repair DNA templates. Through introducing recET-encoded recombinase to increase the probability of homologous recombination, this genome editing method enables the deletion and integration of large DNA fragments, and accomplishes the precise site-directed mutations in the chromosome at a high efficiency. It was successfully applied to modify multiple genes for increasing 1,2-propanediol production. This developed genome editing method will facilitate engineering of native metabolic network or introducing novel pathways in C. glutamicum to synthesize the desired biobased products.

\section{Results}

\section{Optimization of CRISPR-Cas9 system}

To decrease the effect of Cas9 expression on cell viability, cas 9 gene under the control of a weak promoter $\mathrm{P}_{\text {hom }}$ and $r r n B$ terminator was introduced into the wild-type C. glutamicum ATCC 13032 genome at the transposase (Cgl1066) locus, giving rise to strain WT:: $\mathrm{P}_{\text {hom }}$-cas 9 (Fig. 1a and Additional file 1: Figure S1). To test the lethality of Cas9-induced DSB, the sgRNA targeting on upp gene (encoding uracil phosphoribosyltransferase) was designed and inserted into pXMJ19ts under the control of strong promoter $\mathrm{P}_{g l y A}$, resulting in plasmid psgRNA $_{u p p}$. Transformation with psgRNA ${ }_{u p p}$ yielded $\sim 10^{3} \mathrm{cfu} / \mu \mathrm{g}$ plasmid DNA (Fig. 1b), indicating a high escape rate that is inadequate for eliminating wild-type cells. We assumed that the amount of Cas9 protein might be not enough to cleave target DNA effectively. Therefore, a strong promoter $\mathrm{P}_{t u f}$ was used to increase the transcription of chromosomal cas 9 gene. The mRNA level of cas 9 with $\mathrm{P}_{t u f}$ control increased by 4.93 -fold compared to that under the control of $\mathrm{P}_{\text {hom }}$ (Fig. 1c), leading to a decrease in escape rate (Fig. 1b). Then, two ribosome binding sequences (RBS1 and RBS2) with increased translational initiation efficiencies were inserted into the front of the start codon of cas 9 gene, resulting in WT:: $\mathrm{P}_{\text {tuf }}$ RBs1-cas 9 and WT:: $\mathrm{P}_{\text {tuf }}$ RBS2-cas 9 strains (Fig. 1a). This led to the great decreases in the numbers of transformants (Fig. 1b), demonstrating the feasibility of CRISPR/Cas9-based counter-selection. The plasmid-borne Cas 9 expression hindered cell growth of $C$. glutamicum in the previous report [27], therefore, we investigated the effect of chromosome-borne Cas9 expression on cell viability. When these strains harboring different expression cassettes of cas 9 gene were cultivated in BHIS medium, no difference in growth rate was observed (Fig. 1d). Furthermore, the expression level of chromosome-borne Cas9 was visibly lower than those of plasmid-borne IPTG-inducible Cas9 by Western blotting analysis, suggesting that the decreased expression of chromosome-borne Cas9 has little impact on cell growth (Fig. 1e).

To assess the editing efficiencies using sgRNA and different Cas9 expression cassettes for gene deletion in $C$. glutamicum, the upp gene encoding uracil phosphoribosyltransferase was chosen as the target gene. Since its inactivation led to the failure of conversion from 5-fluorouracile (5-FU) to a toxic product for cell growth and generated a 5-FU-resistant phenotype for convenient screening [8]. To delete upp gene, two homologous arms as repair templates were inserted into psgRNA to construct pHAsgRNA ${ }_{u p p}$. When transformed with pHAsgRNA $_{\text {upp }}$ into WT:: $\mathrm{P}_{\text {hom }}$-cas $9,21.79 \pm 5.88 \%$ of the colonies $(7 / 26,4 / 26,6 / 26)$ were confirmed by colony PCR to correctly edit upp gene (Fig. If and Additional file 1: Figure S2) and correspondingly gained the 5-FU resistance (Fig. 1g). For the next round genome editing, nearly all of colonies after an overnight incubation had lost the plasmid pHAsgRNA ${ }_{u p p}$, indicating the convenience of a single-plasmid-based CRISPR-Cas9 system for genome editing in C. glutamicum. When transformed

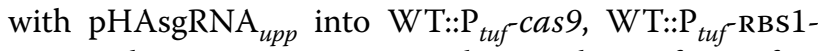
cas 9 and WT:: $\mathrm{P}_{\text {tuf }}$-RBS2-cas9, the numbers of transformants decreased (Fig. 1f); however, the editing efficiency improved from $43.59 \pm 4.44$ to $60.26 \pm 5.88 \%$ (Fig. If and Additional file 1: Figures S3-S5), indicating that the lethal efficiency of CRISPR/Cas9-based counter selection has a significant effect on editing efficiency for gene deletion.

\section{Introduction of RecET increases the editing efficiency of CRISPR-Cas9}

The edited mutants would be generated from doublecrossover HR; therefore, the HR ability of C. glutamicum became a key factor in the editing efficiency of CRISPR/ Cas9 system. To increase the probability of HR occurrence, recET from the defective Rac prophage under the control of inducible $\mathrm{P}_{p r p}$ were introduced into the chromosome at the downstream of cas 9 locus, giving rise to the WT:: $\mathrm{P}_{t u f}$ RBS2-cas9:: $\mathrm{P}_{p r p}$-recET (Fig. 2a and Additional file 1: Figure S6). As the inducible expression of RecET in the presence of propionate, the transformation with pHAsgRNA $A_{\text {upp }}$ produced $2.1 \times 10^{3} \mathrm{cfu} / \mu \mathrm{g}$ plasmid DNA, which was a $70 \%$ increase compared to WT::P tuf cas 9 (Fig. 2b). About $82.05 \pm 8.88 \%$ of selected colonies were identified as the expected deletion of upp by colony PCR (Fig. 2c and Additional file 1: Figure S7). Two RBSs (RBS3 and RBS4) were inserted in the front of the start codon of recET to improve its translation initiation rate. The numbers of obtained colonies showed no significantly difference (Fig. 2d). As expected, editing 


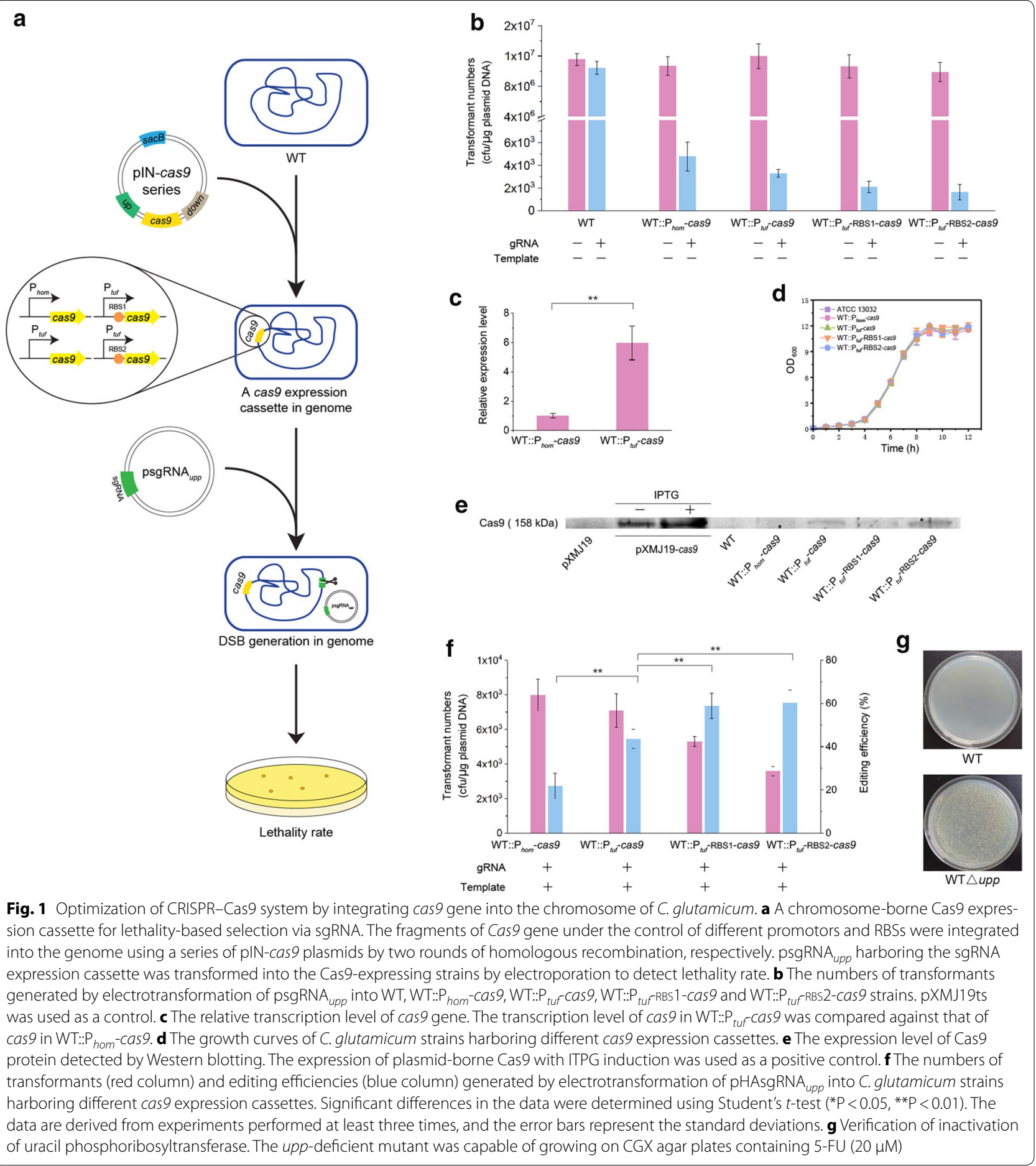

efficiencies improved to $83.33 \pm 2.22 \%$ in WT:: $\mathrm{P}_{t u f}$ RBS2cas9:: $\mathrm{P}_{\text {prp }}-\mathrm{RBS} 3-r e c E T$ and $92.31 \pm 0.00 \%$ in WT:: $\mathrm{P}_{t u f}$

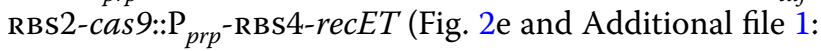
Figures S8, S9), respectively. These results suggested that introduction of recET gene increased the HR efficiency of C. glutamicum, resulting in a high editing efficiency. For convenience, WT:: $\mathrm{P}_{t u f}$-RBS2-cas9:: $\mathrm{P}_{p r p}$-RBS4-recET strain was referred as EDT (Editable Type) in the following text. A detailed experimental protocol of the RecET-assisted CRISPR-Cas9 method had been shown in Additional file 2. 

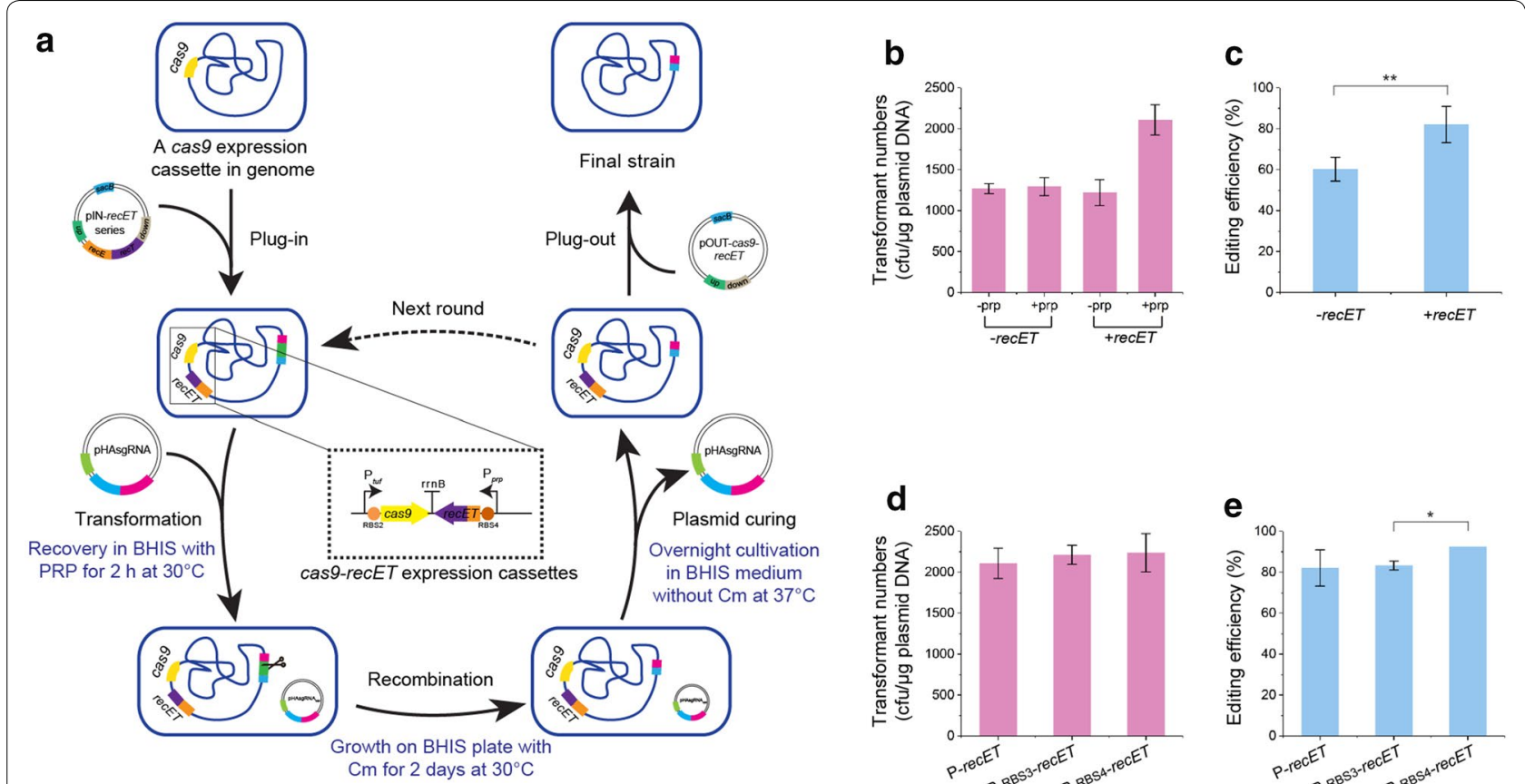

Fig. 2 RecET-assisted CRISPR-Cas9 genome editing in C. glutamicum. a A flow-chart for a single-plasmid-based and RecET-assisted CRISPR-Cas9 editing method in C. glutamicum. RecET gene under control of a propionate-inducible promoter $\mathrm{P}_{\text {prp }}$ was integrated into WT::P $\mathrm{P}_{\text {tuf }}$ RBS $2-$-cas 9 via a series of pIN-recET plasmid by two rounds of homologous recombination. pHAsgRNA was transformed by electroporation into the strain harboring cas9-recET expression cassettes. After the recovery in BHIS supplemented with $0.5 \mathrm{~g} / \mathrm{L}$ sodium propionate (PRP) at $30^{\circ} \mathrm{C}$ for $2 \mathrm{~h}$, cells were spread on chloramphenicol-resistant BHIS plate for 2-day cultivation. The positive colonies screened by colony PCR were subcultured in chloramphenicolfree BHIS medium at $37^{\circ} \mathrm{C}$ overnight for plasmid curing. The edited strain without pHAsgRNA plasmids could be used for the next round of genome editing. Finally, the pOUT-cas9-recET carrying two homologous arms was transformed into the edited strain to plug out the chromosomal cas9-recET expression cassettes by two rounds of homologous recombination to get a final strain. $\mathbf{b}$ The number of transformants generated by electrotransformation of pHAsgRNA into WT::P $P_{\text {tuf }}$ RBS2-cas $9:: P_{\text {prp }}$-recET with or without propionate introduction. c Editing efficiencies under the noninducible or inducible RecET expression conditions. d The number of transformants generated by electrotransformation of pHAsgRNA into

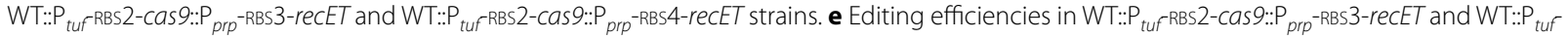
RBS2-Cas9::P prp -RBS4-recET strains. Significant differences in the data were determined using Student's $t$-test $\left({ }^{*} P<0.05,{ }^{* *} P<0.01\right)$. The data are derived from experiments performed at least three times, and the error bars represent the standard deviations

\section{Applicability of CRISPR-Cas9 editing method in C. pekinense}

To determine whether CRISPR-Cas9 system is applicable for other C. glutamicum-related bacteria, Cas9 expression cassette instead of RecET-Cas9 expression cassette was used to integrate into the chromosome due to the low frequency of integrating the $8.5-\mathrm{kb}$ expression cassette of RecET-Cas9 using two rounds of homologous recombination in the chromosome. C. pekinese 1.563 , a lysine producer screened by random mutagenesis was chosen as a candidate strain for genome editing [29]. Two sgRNA targeting argR encoding a regulator and farR encoding a global repressor were designed and used to construct pHAsgRNA ${ }_{\text {argR }}$ and pHAsgRNA farR $_{\text {for delet- }}$ ing these two genes. Transformation with pHAsgRNA $\operatorname{argR}$ produced $2.6 \times 10^{3} \mathrm{cfu} / \mu \mathrm{g}$ plasmid DNA (Fig. 3a). The editing efficiency for argR deletion in C. pekinense 1.563 was $30.3 \%$, which was similar to that in C. glutamicum ATCC 13032 (Fig. 3b and Additional file 1: Figure S10).
And the deletion of far $R$ was accomplished with a transformation efficiency of $2.4 \times 10^{3} \mathrm{cfu} / \mu \mathrm{g}$ plasmid DNA and an editing efficiency of $30 \%$ in C. pekinense 1.563 , respectively (Fig. 3a, c and Additional file 1: Figure S11). Therefore, our results indicated that the CRISPR-Cas9 system could be available for genome editing in other Corynebacterium species.

\section{Gene deletion and integration using RecET-assisted CRISPR-Cas9 method}

The method was further tested for efficiencies of gene deletion at different loci. Firstly, the inactivation of lactate dehydrogenase was performed by deleting $l d h$ gene. Using the same sgRNA as that in the two-plasmidbased CRISPR/Cas9 method [27] for deleting ldh gene and the donor templates with 500 and 1000 bp HAs, $\mathrm{pHA}$ 00sgRNA $l d h$ and pHA1000sgRNA $l d h$ were constructed and transformed into EDT, respectively (Fig. 4a). The editing efficiency with 1000 bp HAs (78.21 $\pm 2.22 \%)$ 


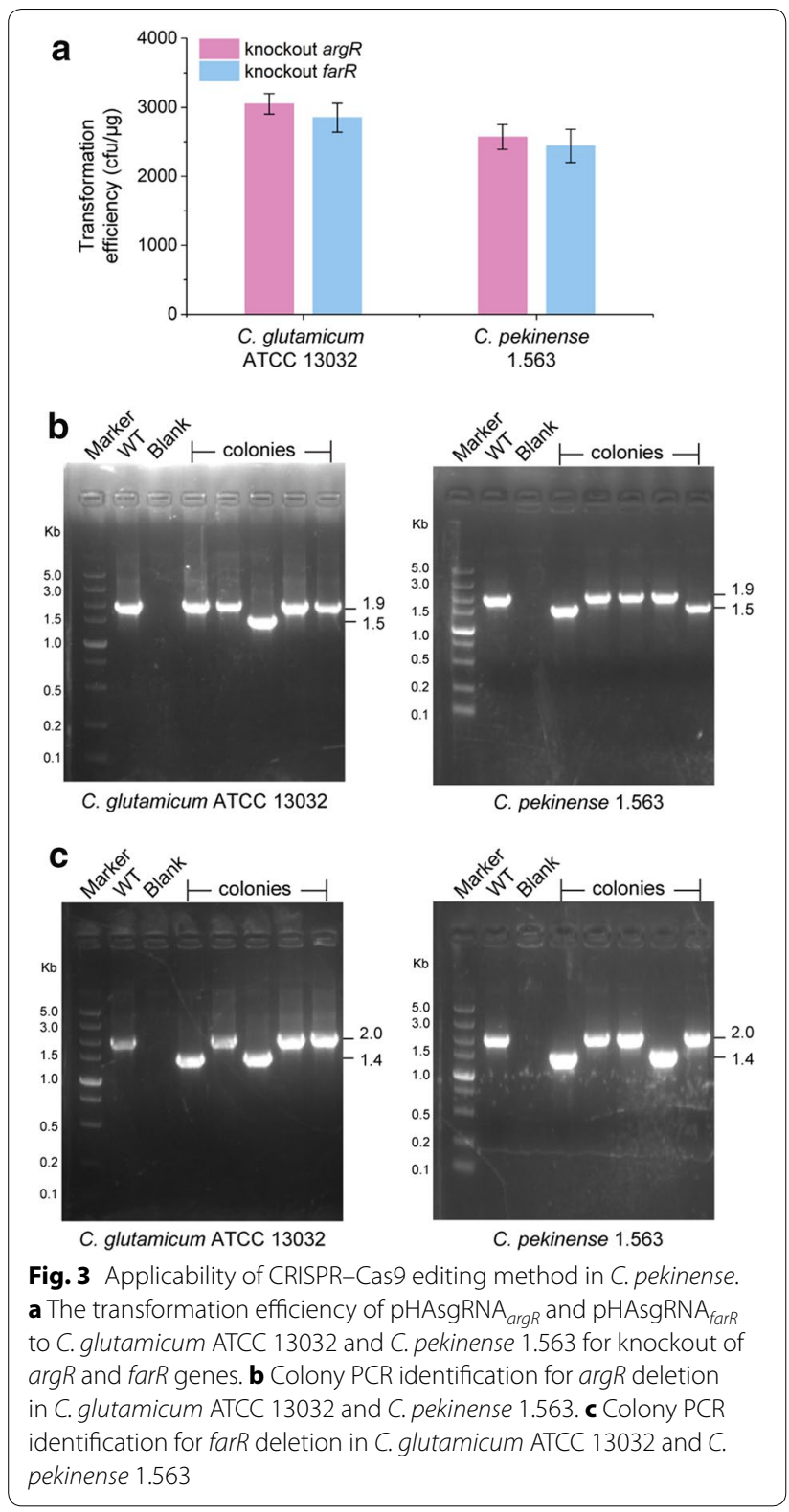

was higher than that with 500 bp HAs $(65.38 \pm 3.85 \%)$ (Additional file 1: Figures S12, S13), which was consistent with the effects of repair arm sizes on gene editing efficiency in the previous report [28]. To validate its efficiencies for deleting the fragments with variant sizes, CGP3, a $220 \mathrm{~kb}$ prophage [30], was chosen as the target to delete 1-, 10-, 20- and 200-kb regions using the same $\operatorname{sgRNA}_{\triangle \mathrm{CGP} 3}$ and different upstream and downstream HAs in EDT (Fig. 4b). The transformation with pHAsgRNA $_{\triangle \mathrm{CGP} 3-1 \mathrm{~kb}}$ into EDT produced $1.29 \times 10^{3} \mathrm{cfu} /$ $\mu \mathrm{g}$ plasmid DNA. Forty-three positive colonies were screened with a deletion efficiency of $55.13 \pm 2.22 \%$ (Fig. 4b and Additional file 1: Figure S14). The transformation efficiencies with $\mathrm{pHAsgRNA}_{\triangle \mathrm{CGP} 3-10 \mathrm{~kb}}$ and pHAsgRNA ${ }_{\triangle \mathrm{CGP} 3-20 \mathrm{~kb}}$ decreased by $43 \%$ and $70 \%$; and the deletion efficiencies of 10 - and 20-kb regions were $35.90 \pm 2.22$ and $26.92 \pm 3.85 \%$, respectively (Additional file 1: Figures S15, S16). Unfortunately, the transformation with $\mathrm{pHAsgRNA}_{\triangle \mathrm{CGP} 3-200 \mathrm{~kb}}$ only yielded $\sim 10$ colonies and the 200-kb deficient mutant was not obtained by colony PCR screening.

For gene insertion, 717-bp gfpmut3a gene as a candidate was inserted in upp, CGP1, CGP2 and CGP3 loci, respectively. The insertion of $g f p$ at $u p p$ locus reached an efficiency of $69.23 \pm 3.85 \%$ (Fig. 4c and Additional file 1: Figure S17). In contrast, the insertion of $g f p$ at CGP1, CGP2 and CGP3 loci only showed editing efficiencies below 10.0\% (Fig. 4c and Additional file 1: Figures S18-S20). Due to the effects of gRNA performance on the cutting effectiveness of the Cas9-sgRNA complex [31-33], we designed nine sgRNAs to target the different PAM sites locating in CGP1. However, the numbers of colonies harboring psgRNA ${ }_{\triangle \mathrm{CGP} 1}$ series vectors were significantly higher than those of colonies harboring

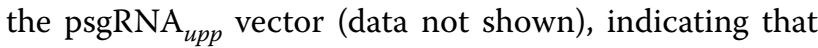
sgRNA performance might be a key factor to affect the editing efficiency of CRISPR-Cas9 system. Using the sgRNA targeting upp locus, a 2.5-kb $\mathrm{P}_{\text {tuf }}$ hom-thrB and 5.7-kb $\mathrm{P}_{\text {tuf }}$ hom-thrB- $\mathrm{P}_{\text {glyA }}$-lysC-thrC expression cassettes were inserted with the editing efficiencies of 7.14 and $2.22 \%$, respectively (Fig. $4 \mathrm{c}$ and Additional file 1: Figure S21). Furthermore, only a positive mutant with a 7.5-kb $\mathrm{P}_{\text {tuf }}$ trpEG- $\mathrm{P}_{g l y A}-\operatorname{trpDC}-\mathrm{P}_{\text {sod }}$-trpBA expression cassette insertion at the $u p p$ locus was obtained through screening 61 transformants by colony PCR (Fig. 4c and Additional file 1: Figure S21). Using two different sgRNAs targeting the same locus between cgl0900 and cgl0901, the 3.6-kb lacZ expression cassette was inserted into the genome with the efficiency of $16.67 \pm 2.22$ and $20.51 \pm 2.22 \%$ (Additional file 1: Figure S22), respectively. Taken together, the differences in genomic locus and the length of inserted fragment had an obvious effect on the insertion efficiency.

\section{Site-directed mutation mediated by RecET-assisted CRISPR-Cas9 method}

To evaluate the efficiency of site-directed mutation, the start codon of pgi gene were mutated from ATG to GTG to downregulate its expression, which led to the increase in the flux distribution towards pentose phosphate pathway [2]. Eleven colonies were identified to be correct edited by PCR amplification and DNA sequencing with an efficiency of $55.0 \%$ (Fig. 5a). When the mutation from CGG to CAG at the PAM was introduced into the repair template, the efficiency for pgi mutation increased to $95 \%$. Then, this method was applied to introduce 


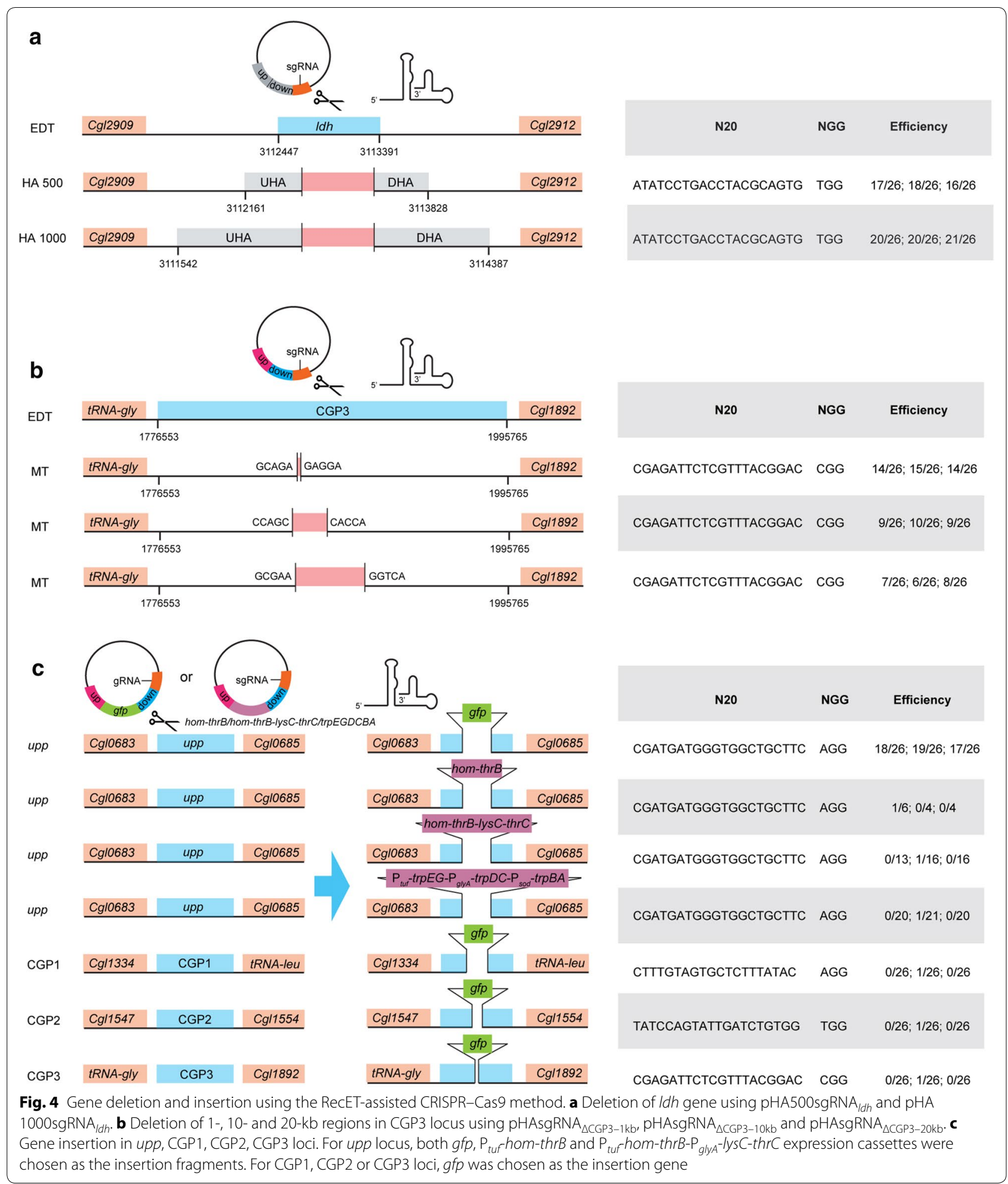

double amino acid mutations of A26V and M31V into the chromosomal ArgB to release the feedback inhibition by $\mathrm{L}$-arginine [2]. The sgRNA targeting the sequence from 90th to 110th bp of $\operatorname{argB}$ gene and HA containing
10 bp mismatches were designed (Fig. 5b). Thirteen colonies were randomly selected for colony PCR identification. Ten colonies contained the total mutated sites in the $\arg B^{*}$ with an editing efficiency of $77 \%$ (Fig. 5b). 


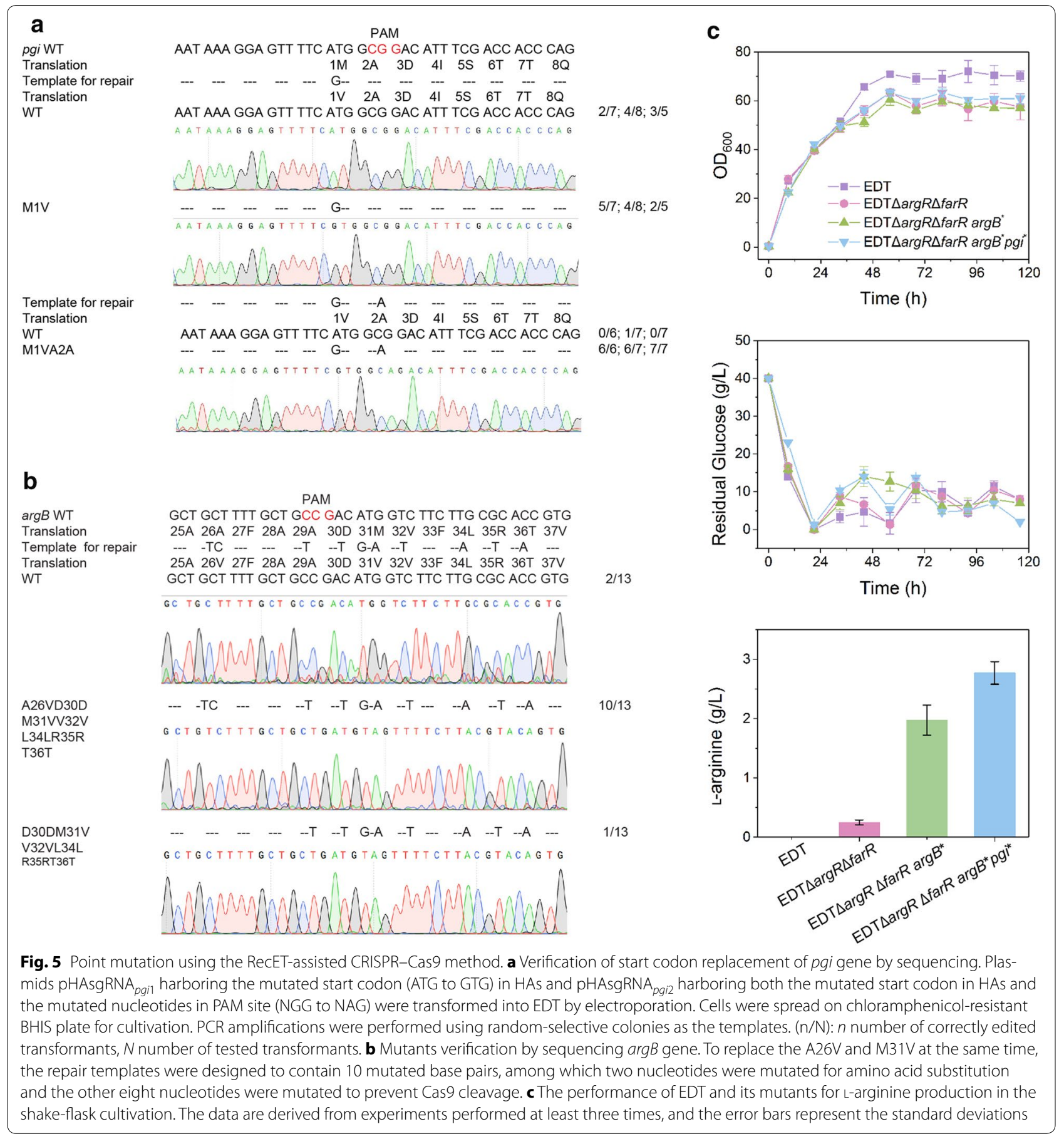

Interestingly, one colony showed eight point mutations without A26V mutation, indicating that the doublecrossover event happened at the downstream sites of A26 to repair the DSB.

Due to the potential instability of plasmid-borne Cas9, we further performed consecutive multiplex gene modifications in EDT to detect the stability of chromosome-borne Cas9. The deletion of $\operatorname{argR}$ showed an editing efficiency of $83.33 \%$ in EDT. The efficiency for farR deletion in EDT $\triangle a r g R$ was $75.00 \%$. Two amino acid mutations of ArgB* was also performed in EDT $\Delta a r g R \Delta f a r R$ with an editing efficiency of $77.06 \%$. Shake flask cultivation showed that EDT $\Delta \operatorname{argR} \Delta$ farRargB* accumulated $1.98 \pm 0.26 \mathrm{~g} / \mathrm{L}$ 
L-arginine. As knockdown of pgi expression in EDT $\Delta \operatorname{argR} \triangle \operatorname{farRarg} B^{*}$, the growth and glucose consumption rates of EDT $\Delta \operatorname{argR} \Delta$ farRargB*pgi $i_{\mathrm{GTG}}$ were comparable to those of other strains (Fig. 5c), and L-arginine production reached $2.77 \pm 0.19 \mathrm{~g} / \mathrm{L}$ (Fig. 5c). These results indicated the stability of chromosome-borne Cas9 for iterative genome editing in C. glutamicum.

\section{Application of RecET-assisted CRISPR-Cas9 method to modify multiple genes for biosynthetic production of 1,2-propanediol}

Corynebacterium glutamicum have been engineered for 1,2-propanediol production by expressing the heterologous genes $m g s A$ encoding methylglyoxal synthase, gld $A$ encoding glycerol dehydrogenase and $y q h D$ encoding alcohol dehydrogenase from E. coli $[34,35]$. Genetic modification strategy for increasing 1,2-propanediol production have been predicted by $i$ CW773 in our previous study [36]. The deletion of $h d p A$ encoding dihydroxyacetone phosphate phosphatase and $l d h$ encoding lactate dehydrogenase could decrease the formation of byproducts, glycerol and lactate, to increase the 1,2-propanediol production (Fig. 6a). Downregulation of gap, pgk and gpmA could decrease the split-flow of glyceraldehyde phosphate and drive more carbon fluxes towards 1,2-propanediol synthesis. Among three genes, $p g k$ encoding phosphoglycerate kinase is essential for growth of $C$. glutamicum on carbon sources requiring glycolysis and gluconeogenesis, and a major control point in the glycolytic pathway during growth on glucose [37]. The effects of decreased Pgk flux on cell metabolism and 1,2-propanediol synthesis were simulated by $i$ CW773 using FBA. The fluxes toward MgsA was significantly enhanced with the decrease in the relative Pgk flux, and the maximum extracellular 1,2-propanediol production was achieved when the relative Pgk flux was decreased by $30 \%$ compared with WT (Fig. 6b). Therefore, these genes were modified by RecET-assisted CRISPR-Cas9-mediated method to regulate the metabolic network for 1,2-propanediol synthesis (Fig. 6a).

Firstly, expression of $m g s A$, gldA, and $y q h D$ genes in an artificial operon under the $\mathrm{P}_{t a c}$ control from the plasmid pXMJ19-mgsA-gldA-yqdD gave rise to $1.69 \pm 0.09 \mathrm{~g} / \mathrm{L}$ 1, 2-propanediol accumulation with a yield of $0.10 \mathrm{~mol} /$ mol in a shake flask cultivation of PT (Fig. 6c). The efficiencies of $h d p A$ and $l d h$ deletion were 66.67 and $50.00 \%$, respectively (Additional file 1: Figures S23, S24). Deletion of $h d p A$ gene resulted in a $94.08 \%$ decrease in the extracellular glycerol accumulation in PT $\Delta h d p A \Delta l d h$ (Fig. 6c). The lactate was almost undetectable in PT $\Delta h d p A \Delta l d h$ that accumulated $4.95 \pm 0.24 \mathrm{~g} / \mathrm{L}$ 1,2-propanediol with a 2.0 -fold increase in the product yield $(0.30 \mathrm{~mol} / \mathrm{mol})$.
The byproduct acetate was accumulated $(2.99 \pm 0.15 \mathrm{~g} / \mathrm{L})$, which was consistent with the previous report [34, 35]. To control the competing pathway for glyceraldehyde phosphate, two weak promoters, $\mathrm{P}_{\text {hom }}$ and $\mathrm{P}_{\text {dapA }}$, were used to construct pHAsgRNA $\mathrm{Phom}_{\text {and }}$ aHAsgRNA $\mathrm{PdapA}$ for the promoter replacements to decrease the transcriptional level of $p g k$ gene. The efficiencies of $\mathrm{P}_{h o m}$ and $\mathrm{P}_{\text {dapA }}$ replacement were 66.67 and $83.33 \%$, resulting in 22.66 and $74.41 \%$ decreases in the mRNA level of $p g k$, respectively (Fig. 6d). Meanwhile, the mRNA level of gapX decreased and the mRNA level of tpiA increased, which contributed to drive the flux toward the 1,2-propanediol synthetic pathway. However, no significant changes were observed in the mRNA levels of fda, gpmA, $g l t A$ and $a c n$. When the mRNA level of pgk decreased, there were no significant differences in the growth and glucose consumption (Fig. 6d). PT $\Delta h d p A \Delta l d h \mathrm{P}_{h o m} . p g k$ and $\mathrm{PT} \Delta h d p A \Delta l d h \mathrm{P}_{\text {dapA }}-p g k$ produced $6.75 \pm 0.46$ and $5.57 \pm 0.36 \mathrm{~g} / \mathrm{L}$ 1,2-propanediol, respectively, which were 36.36 and $12.53 \%$ increase compared to PT $\Delta h d p A \Delta l d h$. Meanwhile, the precursor acetol showed the increasing trend similar to 1,2-propanediol and the byproduct acetate decreased by 25.42 and $13.71 \%$, respectively (Fig. 6c). Finally, the downregulation of $p g k$ improved the product yield to $0.41 \mathrm{~mol} / \mathrm{mol}$ glucose, which was higher than the deletion of $h d p A$ and $l d h$ genes in the previous engineered C. glutamicum [34, 35]. For industrial applications, the cas9-recET expression cassettes were deleted from the chromosome of EDT $\Delta l d h \Delta h d p A \mathrm{P}_{\text {hom }}$-pgk using pOUT-cas9-recET plasmid to obtain a final engineered strain for 1,2-propanediol production (Additional file 1: Figure S25).

\section{Discussion}

CRIPSR-Cas9 systems have been employed to be efficient biotechnology tools for genome editing in many bacteria [18-24]. Two-plasmid-based CRIPSR-Cas9 editing methods available for C. glutamicum require a high transformation efficiency to guarantee the cooccurrence of two plasmids transformation, resulting in their editing efficiencies comparable to those of other methods relying on two rounds of homologous recombination [26-28]. In this study, we developed a RecETassisted CRISPR-Cas9-genome editing method for $C$. glutamicum. This approach employed a single plasmid to transform EDT, which enables transformation and plasmid curing procedures to be more simple and convenient than two-plasmid-based CRISPR-Cas9 method [27, 28]. Introduction of RecET increased the editing efficiency of this method, which is applicable for comprehensive genome editing, such as deletion of large DNA fragments, integration of multiplex gene expression cassettes, 

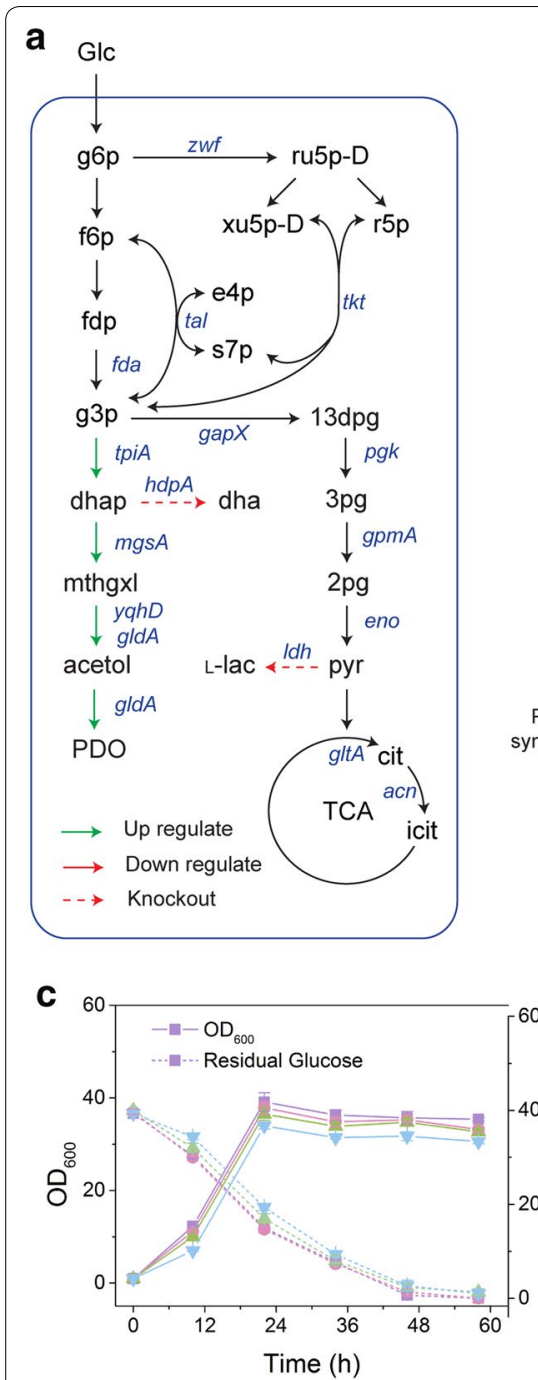

b
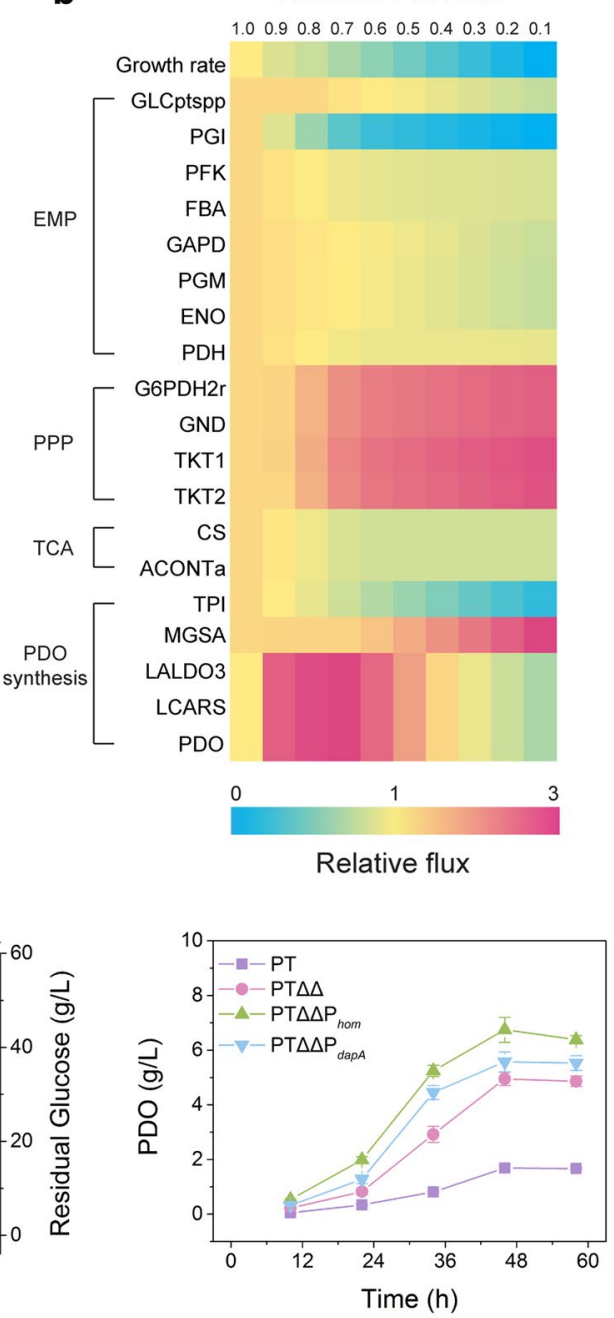
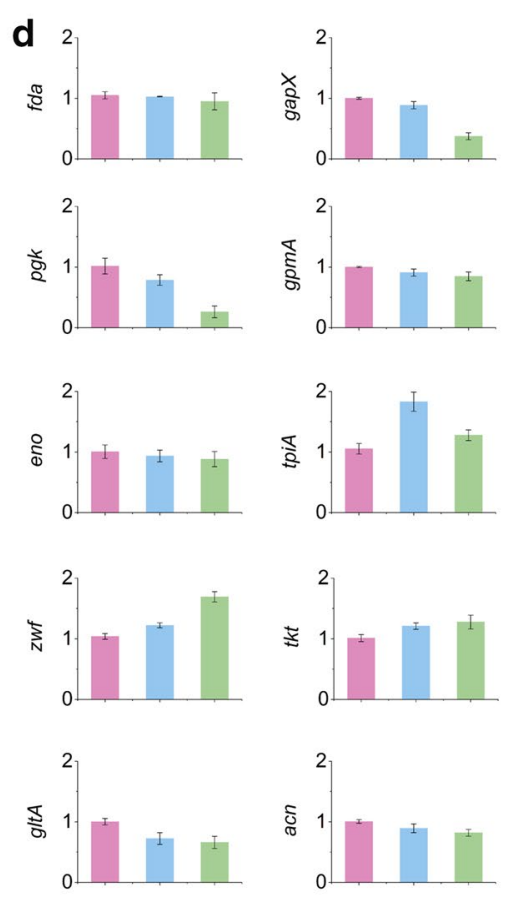

Fig. 6 Multiple gene modifications for 1,2-propanediol production in C. glutamicum using the RecET-assisted CRISPR-Cas9 method. a Strategy of the multiple gene modifications for 1,2-propanediol production in C. glutamicum. b The impact of Pgk flux on cellular metabolism was investigated with decreasing relative Pgk fluxes (between 1.0 and 0.1). X-axis: relative Pgk flux (the ratio of the test Pgk flux to the native Pgk flux). Relative Pgk flux $=1.0$ served as the control (or native GPD flux). Y-axis: relative flux of the pathways (the ratio of the test flux to the native flux). Yellow represents no change, red represents upregulation, and blue represents downregulation of the flux. c Flask cultivation of C. glutamicum strains for 1,2-propanediol (1,2-PDO) production. PT $\Delta \Delta:$ PT $\Delta / d h \Delta h d p A ;$ PT $\Delta \Delta \mathrm{P}_{\text {hom }}$ : PT $\Delta / d h \Delta h d p A \mathrm{P}_{\text {hom }}-p g k ;$ PT $\Delta \Delta \mathrm{P}_{\text {dapA }}$ : PT $\Delta l d h \Delta h d p A \mathrm{P}_{\text {dapA }}$-pgk. The data are derived from experiments performed at least three times, and the error bars represent the standard deviations. $\mathbf{d}$ Relative transcript levels of related genes in the different C. glutamicum strains at the exponential growth phase. Red column: PT $\Delta / d h \Delta h d p A$; blue column: PT $\Delta / d h \triangle h d p A P_{h o m}-p g k$; green column: PT $\triangle / d h \triangle h d p A P_{\text {dapA }}-p g k$

and promoter replacements as well as site-directed mutations.

In two-plasmid-based CRISPR-Cas9 methods, only the decreased expression level of SpCas9 under the control of inducible $\mathrm{P}_{t a c}$ was successful to obtain transformants due to the potential toxicity of SpCas9 on cell viability [26-28]. Even so, the transformants harboring pCas9 grew at a lower rate than the wild-type strain [28]. Moreover, plasmid-borne Cas9 occurred spontaneous mutation, resulting in editing failure [27]. To decrease the copies of cas 9 gene, it was integrated into the chromosome under the control of $P_{\text {tuf. }}$, whose strength was lower than that of $\mathrm{P}_{t a c}$ [38]. Western blotting analysis testified that the expression of chromosome-borne Cas9 maintained at the relative lower level compared to plasmid-borne Cas9, which might be the reason for no impacts of chromosome-borne Cas9 expression on cell growth. Furthermore, no off-target mutations could be detected in C. glutamicum expressing plasmid-borne Cas9 protein by genome sequencing [28], indicating that 
the chromosome-borne Cas9 might generate a very low probability of off target.

The induction of DSB on chromosome by functional Cas9-sgRNA complex is lethal to some bacteria lacking NHEJ pathway like eukaryotes [14, 39, 40]. For C. glutamicum, the co-transformation of CRISPR-Cas9 system with either dsDNA with different HA lengths or ssDNA as editing templates had been testified to be insufficient to repair the DSB in the absence of RecT [26]. The dsDNAs on the plasmids was unable to repair the DSB unless expressing the phage-derived recombinases (RecET) in $E$. coli [18], indicating that homologous recombination efficiency is a key factor that affects the editing efficiency of CRISPR-Cas9 system. However, plasmid-borne dsDNA could repair DSB in C. glutamicum expressing the chromosome-borne Cas9, suggesting that the introduction of DSB by CRISPR-Cas9 system increased the HR of the damaged DNA as reported elsewhere [18]. Introduction of exogenous RecET into C. glutamicum chromosome promoted the frequency of double-crossover event between chromosomal DSB region and repair templates $[8,25,26,41-43]$, which contributed to genome editing mediated by CRISPR-Cas9.

Compared to other CRISPR-Cas9 genome editing methods, our method has some advantages in transformation efficiency and multiple genetic manipulation, indicating a promising application in iterative genome editing of C. glutamicum. A high transformation efficiency is necessary for two-plasmid-based CRISPRCas9-coupled recombineering system to co-transform single-stranded oligodeoxyribonucleotides (ssODN) and Cas9-sgRNA vector into C. glutamicum [26]. A one-step electro-transformation strategy to co-transform pCas9 and pgRNA had been adopted to reduce false positives formation [27]. The low frequency of co-transformation led to less transformants. In contrast, transformation with a single-plasmid harboring sgRNA and HAs produced $10^{3} \mathrm{cfu} / \mu \mathrm{g}$ plasmid DNA. This transformation efficiency could be achieved easily and reproducibly for C. glutamicum at laboratory level, suggesting a universal application of a single-plasmid-based CRISPR-Cas9 system. Moreover, both pCas9 and pgRNA plasmids need to be removed in the absence of antibiotic selection pressure for several subcultures and then be co-transformed together for the next round of genome editing [27]. In contrast, a single-plasmid is easy to be cured by overnight cultivation and another new editing plasmid can be efficiently transformed for the next round of genome editing, indicating the convenience of our method for iterative genome editing. As for gene deletion, the efficiencies of $l d h$ deletion using this method are slightly higher than that using two-plasmid-based CRISPR-Cas9 method (Additional file 1: Table S1) [27]. However, the deletion of 200-kb fragment was unsuccessful, which might be attributed to the decreased efficiency for gene deletion with the increase in gene length as reported previously [9]. Furthermore, CRISPR-Cas9 method relied on a double-crossover event, which simultaneously occurred between the DSB region and donor DNA template after the cleavage of Cas9-sgRNA complex. DSB was generated in the middle part of 200-kb fragment, while the strand exchange of HR occurred between the two sides of 200-kb fragment and plasmid-borne HAs. Therefore, a long spacing between DSB and HAs gave rise to a rare frequency of HR relying on a double-crossover event. However, TCCRAS method could achieve the deletion of 179.8-kb fragment in our previous report, due to a little high probability in the successive occurrence of a singlecrossover event between the chromosomal homologous regions and plasmid-borne HAs under the screening pressure. Despite the numbers of lac $Z$ insertion transformants (13 colonies) obtained by this method were higher than those (4 colonies) screened by two-plasmidbased CRISPR-Cas9 method, the insertion efficiency using this method was relatively low. It attributed to the differences in transformation efficiency and lethality of Cas9 between two methods. A large number of colonies were obtained as transformation with a single plasmid compared to the co-transformation with two plasmids. Meanwhile, the abundance of chromosomal-borne Cas9 maintained at a relatively lower level than that of plasmid-borne Cas9, resulting in the low lethality of chromosomal-borne Cas9 and the high numbers of false positive colonies. Furthermore, the integration efficiencies mediated by CRISPR-Cas9 at different chromosomal position in $E$. coli were variable due to the differences of cutting effectiveness of the Cas9-sgRNA complex [44]. It has also been demonstrated that different sgRNA can generate a great impact on editing efficiency in C. glutamicum. Therefore, the sgRNA less than $60 \%$ GC content is recommended to design for increasing the editing efficiency of CRISPR-Cas9 system in C. glutamicum [28].

\section{Conclusions}

In this study, we developed a RecET-assisted CRISPRCas9 genome editing method for C. glutamicum. This method took advantage of the chromosome-borne Cas9 to reduce the instability of plasmid harboring Cas9 and utilized RecET to increase the frequency of homologous recombination. Transformation efficiency was improved by using a single-plasmid harboring a sgRNA and repair template, resulting in high efficiencies in gene deletion, insertion and site-specific mutation. In silico modelpredicted target genes were modified using this method, 
and the final engineered strain produced $6.75 \pm 0.46 \mathrm{~g} / \mathrm{L}$ 1, 2-propanediol. This method will boost the metabolic engineering of C. glutamicum to produce the desired biobased products from renewable biomass.

\section{Methods}

Bacterial strains, plasmids and cultivation conditions Corynebacterium glutamicum ATCC 13032 (American Type and Culture Collection, Manassas, VA, USA) was used as the parental strain for genome editing. The $E$. coli strain EC135 [45] was used as the cloning host for plasmid construction. The pK18mobsacB was used for the introduction of cas 9 and recET genes into the chromosome. The pXMJ19ts with a temperature-sensitive replication origin [46] was applied to express sgRNA cassette and carry the DNA repair template for gene deletion, insertion and site-directed mutation. All of the strains and plasmids used in this study are listed in Additional file 1: Tables S2 and S3. E. coli strains were cultured in Luria-Bertani medium at $37^{\circ} \mathrm{C}$. C. glutamicum strains were cultured in brain heart infusion medium at $30{ }^{\circ} \mathrm{C}$. When necessary, antibiotics were added at the following concentrations: $50 \mu \mathrm{g} / \mathrm{mL}$ kanamycin or $20 \mu \mathrm{g} / \mathrm{mL}$ chloramphenicol for $E$. coli and $25 \mu \mathrm{g} / \mathrm{mL}$ kanamycin, $10 \mu \mathrm{g} /$ $\mathrm{mL}$ chloramphenicol for C. glutamicum.

\section{DNA manipulations}

DNA manipulations were performed using the standard cloning techniques [47], and all primers for plasmids construction are shown in Table S4. Polymerase chain reaction (PCR) amplifications were performed with Q5 High-Fidelity DNA Polymerase (NEB) and Taq DNA Polymerase (Tiangen) for cloning and colony PCR identification, respectively. Gibson Assembly were conducted with NEBuilder Master Mix (NEB). DNA gel Extraction Kit (Tiangen) was used to concentrate the DNA fragment and NanoDrop 2000 UV-Vis Spectrophotometer (Thermo Scientific) was used to determine the concentration of DNA fragment for Gibson Assembly [48].

\section{Insertion of Cas9 and RecET expression cassettes into chromosome}

To introduce cas 9 gene into the chromosome, the cas 9 gene was amplified using $\mathrm{pCas}$ as the template with primers P5/P6. $\mathrm{P}_{\text {hom }}$, the upstream homologous arms (HA), and downstream HA were amplified using C. glutamicum ATCC 13032 genome as template with primers P3/ $\mathrm{P} 4, \mathrm{P} 1 / \mathrm{P} 2$ and $\mathrm{P} 9 / \mathrm{P} 10$, respectively. The terminator $r r n$ was amplified from pXMJ19 with primers P7/P8. These fragments together with linearized pK18mobsacB were purified and recovered for Gibson assembly to construct pIN-P hom-cas . To construct pIN-P $\mathrm{P}_{\text {tuf }}$ cas 9 , pIN-P tuf RBS1-cas 9 and pIN-P ${ }_{t u f}$ RBS2-cas9, the same procedures were applied using the indicated primers shown in Additional file 1: Table S4. Then, pIN-P hom $^{-c a s 9, \text { pIN-P }}$ tuf cas 9 , pIN-P tuf $^{\mathrm{RBS} 1-c a s 9}$ and pIN-P tuf $\mathrm{RBS} 2$-cas 9 were transformed to the competent cells of C. glutamicum ATCC 13032 by electroporation, respectively. Screening for the first and second recombination events were performed as described previously [7]. Kanamycin-sensitive and sucrose-resistant clones were verified by colony PCR and DNA sequencing to obtain WT:: $\mathrm{P}_{\text {hom }}$-cas 9 , WT::: $\mathrm{P}_{\text {tuf }}$ cas9, WT:: $\mathrm{P}_{\text {tuf }}$ RBs1-cas9, WT:: $\mathrm{P}_{\text {tuf }}$-RBS2-cas9 strains (Additional file 1: Figure S1).

To integrate recET gene into the chromosome, E. coli W3110 genome was used as a template to amplify recET(anti) with primers P33/P34. WT:: $\mathrm{P}_{\text {hom }}$-Cas9 genome was used as a template to amplify cas_tail_rrnB with primers P31/P32. WT:: $\mathrm{P}_{\text {hom }}$-Cas9 genome was used as a template to amplify $\mathrm{P}_{p r p}$ (anti) and down HA with primers P35/P36 and P37/P38. These fragments together with linearized pK18mobsacB were purified and recovered for Gibson assembly to construct pIN-P prp $_{\text {-recET }}$. The same procedures were performed to construct pIN$\mathrm{P}_{p r p}$-RBS3-recET and pIN-P $\mathrm{P}_{p r}$-RBS4-recET using the indicated primers in Additional file 1: Table S4. Then, pIN-P $\mathrm{P}_{p}-r e c E T, \quad \mathrm{pIN}-\mathrm{P}_{p r p}-\mathrm{RBS} 3-r e c E T$ and $\mathrm{pIN}-\mathrm{P}_{p r p^{-}}$ RBS4-recET were transformed to WT:: $\mathrm{P}_{t u f}$ RBS2-cas9 by electroporation. Screening for the first and second recombination events were performed as described previously [7]. Kanamycin-sensitive and sucrose-resistant clones were verified by colony PCR and DNA sequencing to obtain WT:: $\mathrm{P}_{\text {tuf }}$ - RBS2-cas9:: $\mathrm{P}_{p r p}$-recET, WT:: $\mathrm{P}_{t u f}$ RBS2cas $9:: \mathrm{P}_{p r p}-\mathrm{RBS} 3-$ recET and WT:: $\mathrm{P}_{\text {tuf }} \mathrm{RBS} 2$-cas $9:: \mathrm{P}_{p r p}{ }^{-}$ RBS4-recET strains (Additional file 1: Figure S6).

\section{Construction of sgRNA expression plasmids}

To construct sgRNA expression plasmid, the 20 bp spacer sequence specific for each target was synthesized in primers and the sgRNA scaffold was amplified by PCR using pTargetF as the template. The $\mathrm{P}_{g l y A}$ for sgRNA transcription and the upstream and downstream HAs for DSB repair were amplified using genome of C. glutamicum ATCC 13032 as the template. The pXMJ19 was used as a template to amplify mutated pXMJ19ts using primers P11/P12. The PCR product was digested with $D p n \mathrm{I}$ and transformed into E. coli to obtain pXMJ19ts [49]. All the fragments and the linearized pXMJ19ts as the backbone were fused by Gibson Assembly [48], and followed by transformation in EC135 [45].

To construct $\mathrm{pHAsgRNA}_{u p p}$, $\mathrm{pHAsgRNA}_{n c} \mathrm{pHAsgRNA}$ ${ }_{\text {argR }}$, $\mathrm{pHAsgRNA}_{\text {farR }}, \mathrm{pHAsgRNA}_{\text {argB10}}, \mathrm{pHAsgRNA}_{\text {argB3 }}$, phom-thrBHAsgRNA ${ }_{\text {upp }}, \mathrm{pHAsgRNA}_{\text {gi } i 1}, \mathrm{pHAsgRNA}_{\text {pgi }}$, pHA500sgRNA $_{l d h}, \quad$ pHA1000sgRNA $_{l d h}, \quad$ pHAsgRNA ${ }_{\triangle \mathrm{CGP} 3-1 \mathrm{~kb}}$, pHAsgRNA ${ }_{\triangle \mathrm{CGP} 3-10 \mathrm{~kb}}, \mathrm{pHAsgRNA} \mathrm{CCGP3-20kb}$, p $f f$ HAsgRNA $_{\text {upp }}, \quad \mathrm{p} g f p \mathrm{HAsgRNA}_{\mathrm{CGP}}, \quad \mathrm{p} g f p \mathrm{HAsgRNA}$ 


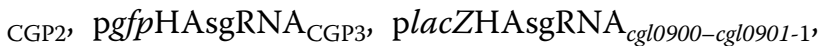
placZHAsgRNA cglo900-cglo901-2, $_{\text {pHAsgRNA }}$ hdpA, pHAsg $\mathrm{RNA}_{\triangle \mathrm{P} p g k: P h o m}$, and pHAsgRNA ${ }_{\triangle \mathrm{P} p g k: P d a p A}$, the abovementioned procedures were conducted using the indicated primers in Additional file 1: Table S4. The integrity of all plasmids constructed in this study was confirmed by sequence analysis.

\section{Gene editing using the RecET-assisted CRISPR-Cas9 method}

We established a single-plasmid-based approach for CRISPR-assisted genome editing in C. glutamicum. The competent cells of C. glutamicum harboring the chromosomal cas9-recET expression cassettes were prepared as previously described [50]. $100 \mathrm{ng}$ of the pHAsgRNA were transformed by electroporation into the competent cells, and then cells were recovered in the BHIS medium supplementing with $0.5 \mathrm{~g} / \mathrm{L}$ sodium propionate for RecET induction at $30{ }^{\circ} \mathrm{C}$ for $2 \mathrm{~h}$. After that, cells were spread on BHIS plate containing $10 \mu \mathrm{g} / \mathrm{mL}$ chloramphenicol to incubate for 2 days. The transformants were screened by colony PCR with corresponding primers and verified by DNA sequencing. c.f.u. was normalized for per $1 \mu \mathrm{g}$ DNA to calculate the transformation efficiency. The editing efficiency is defined as the ratio of correctly edited colonies to the total tested colonies [9], and calculated using the following formula:

Editing efficiency $=$ Number of correctly edited colonies/ Number of tested colonies.

After screening the colonies with correct editing, cells were incubated in BHIS medium at $37{ }^{\circ} \mathrm{C}$ for overnight cultivation and plated to BHIS plates without chloramphenicol to cure the plasmid pHAsgRNA. To determine the curing efficiency, colonies were transferred to LB agar plates with and without chloramphenicol for screening the chloramphenicol sensitive colonies. Due to the high efficiency of plasmid curing, colonies could be used for the next round of RecET-assisted CRISPR-Cas9 genome editing without chloramphenicol sensitivity test.

\section{Plug out cas9-recET expression cassettes from chromosome}

For plug out the chromosomal cas9-recET expression cassettes, the upstream and downstream homologous arms (HA) of chromosome-borne cas9-recET expression cassettes were amplified using P188/P189 and P190/ P191, and then ligated into the pK18mobsacB by Gibson Assembly with NEBuilder Master Mix (NEB) to construct pOUT-cas9-recET. Subsequently, the pOUT-cas9-recET plasmid was transformed into the edited strains to delete cas9-recET expression cassettes by two rounds of homologous recombination to get a final strain.

\section{CRISPR/Cas9-assisted genome editing in C. pekinense}

A lysine-producing C. pekinense 1.563 screened by random mutagenesis was used to verify the application of the CRISPR/Cas 9 system. To insert cas 9 expression cassette, the non-replicated pIn- $\mathrm{P}_{t u f}$-RBS2-cas 9 plasmid was electroporated into the competent cells of $C$. pekinense 1.563 to obtain $1.563:: \mathrm{P}_{\text {tuf }}$-RBS2-cas9 strain by two rounds of recombination events. To delete $\arg R$ and farR genes, $100 \mathrm{ng}$ of the pHAsgRNA ${ }_{\text {argR }}$ and pHAsgRNA ${ }_{\text {farR }}$ plasmids were then transformed by electroporation into 1.563:: $\mathrm{P}_{\text {tuf }} \mathrm{RBS2}$-cas9, respectively. Cells were recovered in the resuscitation medium, spread on BHIS plates supplemented with $10 \mu \mathrm{g} / \mathrm{mL}$ chloramphenicol and then incubated for 2 days. The procedures for transformant screening and plasmid curing were performed as above described in C. glutamicum ATCC 13032.

\section{RNA preparation and quantitative RT-PCR}

In order to determine the level of cas 9 of two cas9expressing strain, i.e. WT:: $\mathrm{P}_{\text {hom }}$-cas 9 and $\mathrm{WT}:: \mathrm{P}_{\text {tuf }}$ cas 9 , quantitative reverse transcription PCR (qRT-PCR) was performed using total RNA samples. Total RNA was isolated from the cells with the RNAprep Pure Cell/Bacteria Kit (Tiangen, China). The cDNA from approximately $300 \mathrm{ng}$ of RNA was prepared using the specific primers listed in Additional file 1: Table S4 and the FastQuant RT Kit (Tiangen, China). The $r p o B$ gene, which encodes RNA polymerase $\beta$ subunit, was used as the reference gene [51]. The primers used for RT-PCR were listed in Additional file 1: Table S4. The qRT-PCR was performed with the GoTaq qPCR master mix (Promega, USA) in a $20-\mu \mathrm{L}$ mixture using the LightCycler ${ }^{\circledR} 96$ Real-Time PCR System (Roche, Switzerland). The data analyses were performed using the LightCycler ${ }^{\circledR} 96$ software (Roche, Switzerland).

\section{Western blotting}

Corynebacterium glutamicum cells were resuspended in $1 \mathrm{~mL}$ of phosphate-buffered saline (PBS) and disrupted using an Ultrasonic Cell Disruptor. The supernatants were collected by centrifugation at $12,000 \mathrm{rpm}$ at $4{ }^{\circ} \mathrm{C}$ for $30 \mathrm{~min}$. Protein samples were separated by Sodium dodecyl sulfate-polyacrylamide gel electrophoresis (SDSPAGE) using a $10 \%$ running gel and $5 \%$ stacking gel and transferred to a nitrocellulose membrane (PVDF) by electroblotting. The proteins were probed with rabbit 
polyclonal antibody against Cas9. The blots were visualized with a peroxidase-coupled goat anti-rabbit secondary antibody and an ECL color development reagent (GE, USA).

\section{Modification of L-arginine synthetic network in $C$. glutamicum}

The strain WT:: $\mathrm{P}_{t u f}$ RBS2-cas9:: $\mathrm{P}_{p r p}-\mathrm{RBS} 4-r e c E T$ (EDT) was used to construct the engineered strains for L-arginine production. The plasmid $\mathrm{pHAsgRNA}_{\text {argR }}$ and $\mathrm{pHAsgRNA}_{\text {farR }}$ were iteratively transformed by electroporation to delete $\arg R$ and farR genes encoding two transcriptional regulator as the above-described procedure, resulting in the strains EDT $\triangle a r g R$ and EDT $\Delta a r g R \Delta$ farR. pHAsgRNA ${ }_{\text {argB } 10}$ was transformed by electroporation into the strain EDT $\Delta a r g R \Delta$ farR for site-directed mutation of chromosomal $\arg B$ to release the feedback inhibition by L-arginine. The plasmid pHAsgRNA $_{p g i 2}$ was transformed by electroporation into the strain EDT $\Delta \operatorname{argR} \Delta$ farRargB* ${ }^{*}$ to change the start codon ATG to GTG for pgi knockdown, resulting in the strain ET $\Delta \operatorname{argR} \Delta$ farRargB* pgi $_{\mathrm{GTG}}$.

\section{Modification of 1,2-propanediol synthetic network in C. glutamicum}

The strain WT:: $\mathrm{P}_{t u f}$ RBS2-cas9:: $\mathrm{P}_{p r p}-\mathrm{RBS} 4-r e c E T$ (EDT) was used to construct the engineered strains for 1,2-propanediol production. The plasmid $\mathrm{pHAsgRNA}_{l d h}$ and pHAsgRNA $_{h d p A}$ were iteratively transformed by electroporation to delete $l d h$ and $h d p A$ as the above-described procedure, resulting in the strain EDT $\Delta l d h \Delta h d p A$. pHAsgRNA ${ }_{\text {Phom }}$ and pHAsgRNA $A_{\text {PdapA }}$ were transformed by electroporation into the strain EDT $\Delta l d h \Delta h d p A$ separately for promoter replacement of $p g k$ to increase the flux towards 1,2-propanediol, generating the strains EDT $\Delta l d h \Delta h d p A \mathrm{P}_{h o m}-p g k$ and EDT $\Delta l d h \Delta h d p A \mathrm{P}_{d a p A}-p g k . \quad \mathrm{pXMJ19-mgsA-gldA-yqhD}$ was transformed by electroporation into the strains EDT, EDT $\Delta l d h \Delta h d p A$, EDT $\Delta l d h \Delta h d p A \mathrm{P}_{h o m}-p g k$ and EDT $\Delta l d h \Delta h d p A \mathrm{P}_{d a p A}-p g k$, giving rise to the strains PT, PT $\Delta l d h \Delta h d p A, \quad \mathrm{PT} \Delta l d h \Delta h d p A \mathrm{P}_{h o m}-p g k$ and $\mathrm{PT} \Delta l d h \Delta h d p A \mathrm{P}_{d a p A^{-}} p g k$, respectively. To obtain a final engineered strain for 1,2-propanediol production, the plasmid pOUT-cas9-recET was transformed into the strain EDT $\Delta l d h \Delta h d p A \mathrm{P}_{h o m}-p g k$ by electroporation to plug out the cas9-recET expression cassette in the chromosome to get a final engineered 1,2-PDO strain.

\section{Shake flask cultivation}

For L-arginine fermentation, C. glutamicum strains were precultured in the CGIII seed medium [52] at $30{ }^{\circ} \mathrm{C}$ and $220 \mathrm{rpm}$ until the $\mathrm{OD}_{600}$ reached 10 . One milliliter of seed culture was inoculated in 500-mL baffled shake flasks containing 30 mL CGXII medium supplemented with $40 \mathrm{~g} / \mathrm{L}$ glucose and $2 \mathrm{~g} / \mathrm{L}$ yeast extract. The cells were grown at $30{ }^{\circ} \mathrm{C}, 220 \mathrm{rpm}$ and the $\mathrm{pH}$ was maintained at $7.0-7.2$ by supplementation with ammonia.

For 1,2-propanediol fermentation, C. glutamicum strains were precultured in CGIII seed medium. The cells of an overnight preculture were inoculated into $40-\mathrm{mL}$ fermentation cultures in 500-mL baffled flasks by transferring appropriate volume for a final optical density of 1.0. Fermentation medium contained $(\mathrm{g} / \mathrm{L})$ : glucose 40 , yeast extract $2, \mathrm{MgSO}_{4} \cdot 7 \mathrm{H}_{2} \mathrm{O} 0.25,\left(\mathrm{NH}_{4}\right)_{2} \mathrm{SO}_{4} 10$, $\mathrm{KH}_{2} \mathrm{PO}_{4} 1, \mathrm{~K}_{2} \mathrm{HPO}_{4} 1, \mathrm{FeSO}_{4} \cdot 7 \mathrm{H}_{2} \mathrm{O} 0.01, \mathrm{MnSO}_{4} \cdot \mathrm{H}_{2} \mathrm{O}$ $0.01, \mathrm{ZnSO}_{4} \cdot 7 \mathrm{H}_{2} \mathrm{O} 0.001, \mathrm{CuSO}_{4} 0.0002, \mathrm{NiCl}_{2} \cdot 6 \mathrm{H}_{2} \mathrm{O}$ 0.00002 , biotin 0.0002 , thiamine 0.0002 and $3-(N$-morpholino) propanesulfonic acid (MOPS) 42. The cells were grown at $30{ }^{\circ} \mathrm{C}$ and $160 \mathrm{rpm}$. The expressions of $m g s A$, $\operatorname{gld} A$, and $y q h D$ genes were induced by adding $1 \mathrm{mM}$ isopropyl- $\beta$-D-thiogalactopyranoside (IPTG) at $6 \mathrm{~h}$. The $\mathrm{pH}$ was maintained at $7.0-7.2$ by supplementation with ammonia.

\section{Analytical methods}

The glucose and lactate concentrations were assayed using an SBA-40D biosensor analyzer (Institute of Biology of Shandong Province Academy of Sciences, Shandong, China). The cell concentration was determined by measuring the absorbance at $600 \mathrm{~nm}\left(\mathrm{OD}_{600}\right)$ using a spectrophotometer (V-1100D; Mapada Instruments, Shanghai, China). To quantify L-arginine, high-pressure liquid chromatography (HPLC) was performed using an Agilent 1260 series chromatography system equipped with a ZORBAX Eclipse AAA column $\left(4.6 \mathrm{~mm} \times 150 \mathrm{~mm}, 5 \mu \mathrm{m}\right.$; Agilent) at $40{ }^{\circ} \mathrm{C}$. Fluorescence detection was carried out after automatic pre-column derivatization with $O$-phthalaldehyde (OPA) using a variable wavelength detector (VWD) at $338 \mathrm{~nm}$ according to the instruction manual. The 1,2-propanediol, acetol, glycerol and acetate in the shake flask were analyzed using a Gas Chromatograph Mass Spectrometer (GCMSQP2010 Ultra, Shimadzu, Japan) connected to an AOC20i Auto-sample using a TG-WAXMS (length: $30 \mathrm{~m}$; I.D.: $0.25 \mathrm{~mm}$; film: $0.25 \mu \mathrm{m}$ ) (Thermo Scientific, USA). The samples were directly diluted 1:10 with methanol and centrifuged by $12,000 \times g$ for injection. The operating set up followed as the previous report [35]. The concentrations of 1,2-propanediol, acetol, glycerol and acetate were determined according to a calibration curve with an external standard. The peaks were identified by retention time and quantified using the intensity of one specific $m / z$ value. 


\section{Additional files}

Additional file 1: Figure S1. Colony PCR verification of cas 9 integration. Figure S2. Colony PCR verification of upp deletion in WT::P hom $_{\text {- }}$ - $a$ S9. Figure S3. Colony PCR verification of upp deletion in WT:: $P_{\text {tuf }}$ cas 9 . Figure S4. Colony PCR verification of upp deletion in WT::P tuf RBS1-cas9. Figure S5. Colony $P C R$ verification of upp deletion in WT::P $P_{\text {tuf }}$ RBS2-cas9. Figure S6. Colony PCR verification of recET integration. Figure S7. Colony PCR verification of upp deletion in WT::P $P_{\text {tuf }}$ RBS2-cas $9: \mathrm{P}_{\text {prp }}$-recET. Figure $\mathbf{S 8 .}$ Colony PCR verification of upp deletion in WT::P tuf $\mathrm{RBS} 2$-cas $9: \mathrm{P}_{\text {prp }}$-RBS3recET. Figure S9. Colony PCR verification of upp deletion in WT::P $\mathrm{P}_{\text {tuf }} \mathrm{fBS} 2-$ cas9:: $P_{\text {prp }}$-RBS4-recET (EDT). Figure S10. Colony PCR verification of arg $R$ deletion in EDT. Figure S11. Colony PCR verification of farR deletion in EDT $\triangle \arg R$. Figure S12. Colony PCR verification of Idh deletion via pHA500sgRNA $A_{\text {Id }}$ in EDT. Figure S13. Colony PCR verification of Idh dele-

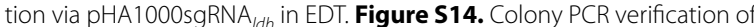
$1-\mathrm{kb}$ fragment deletion at the CGP3 locus in EDT. Figure S15. Colony PCR verification of 10-kb fragment deletion at the CGP3 locus in EDT. Figure S16. Colony PCR verification of 20-kb fragment deletion at the CGP3 locus in EDT. Figure S17. Colony PCR verification of gfp and hom-thrB insertion at the upp locus in EDT. Figure S18. Colony PCR verification of gfp insertion at the CGP1 locus in ET. Figure S19. Colony PCR verification of $g f p$ insertion at the CGP2 locus in EDT. Figure S20. Colony PCR verification of gfp insertion at the CGP3 locus in EDT. Figure S21. Colony PCR verification of $\mathrm{P}_{\text {tuf }}$ hom-thrB, $\mathrm{P}_{\text {tuf }}$ hom-thrB-P $\mathrm{P}_{\text {gly }}-\mathrm{l}_{\mathrm{C}} \mathrm{ySC}$-thrC and $\mathrm{P}_{\text {tuf }}$ trpEG-P $\mathrm{P}_{\text {gly }} \mathrm{A}^{-}$ $\operatorname{trpDC}-\mathrm{P}_{\text {sod }}$-trpBA insertions at the upp locus in EDT. Figure S22. Colony $P C R$ verification of lac $Z$ fragment insertion into the genomic locus between cg/0900 and cg/0901. Figure S23. Colony PCR verification of Idh deletion in EDT. Figure S24. Colony PCR verification of $h d p A$ deletion in EDT $\triangle / d h$. Figure S25. Colony PCR verification of plug out of cas9-recET expression cassette in EDT $\triangle / d h \triangle h d p A P_{h o m}-p g k$. Table S1. Editing efficiencies using different genome editing methods in C. glutamicum. Table S2. Strains used in this study. Table S3. Plasmids used in this study. Table S4. Primers used in this study.

Additional file 2: Standard protocol of a RecET-assisted CRISPR-Cas9 genome editing in Corynebacterium glutamicum.

\section{Abbreviations}

TCCRAS: toxin counter-selectable cassette regulated by an antitoxin switch; CRISPR: clustered regularly interspaced short palindromic repeat; sgRNA: single guide RNA; DSB: double-strand break; PAM: protospacer adjacent motif; NHEJ: nonhomologous end joining; HR: homologous recombination; ssDNA: single-stranded DNA; RBS: ribosome binding sequences; EDT: editable type; ssODN: single-stranded oligodeoxyribonucleotides; PCR: polymerase chain reaction; HA: homologous arm; UHA: upstream homologous arm; DHA: downstream homologous arm; qRT-PCR: quantitative reverse transcription PCR; PBS: phosphate-buffered saline; $\mathrm{OD}_{600}$ : optical density at $600 \mathrm{~nm}$; HPLC: high-pressure liquid chromatography; OPA: O-phthalaldehyde; VWD: variable wavelength detector; GCMS: gas chromatograph mass spectrometer; IPTG: isopropyl- $\beta$-D-thiogalactopyranoside.

\section{Authors' contributions}

TW and $Y Z$ conceived the study. TW, YZ, BW, QH and YZ designed the study. $B W, Q H, Y Z, R S, X C$ and $X S$ performed the experiments. BW, $Q H, Y Z$ and $Y Z$ performed all data analysis. YZ, BW and TW wrote the paper. All authors read and approved the final manuscript.

\section{Author details \\ ${ }^{1}$ CAS Key Laboratory of Pathogenic Microbiology and Immunology, Institute of Microbiology, Chinese Academy of Sciences, Beijing 100101, China. ${ }^{2}$ University of Chinese Academy of Sciences, Beijing 100049, China. ${ }^{3}$ Beijing Zhongke Eppen Biotech Co., Ltd, Beijing 100085, China. ${ }^{4}$ Savaid Medical School, University of Chinese Academy of Sciences, Beijing 100049, China.}

\section{Acknowledgements}

The authors would like to thank Guoxia Liu for assistance with the GC-MS analysis and Tong Zhao for assistance with the flow cytometry analysis.
Competing interests

The authors declare that they have no competing interests.

Ethics approval and consent to participate

Not applicable.

\section{Consent for publication}

Not applicable.

Availability of data and materials

Not applicable.

\section{Funding}

This work was supported by Grants from the Science and Technology Service Network Initiative of the Chinese Academy of Sciences (KFJ-STS-QYZD-047 and KFJ-EW-STS-078) and National Natural Science Foundation of China (31100074).

\section{Publisher's Note}

Springer Nature remains neutral with regard to jurisdictional claims in published maps and institutional affiliations.

Received: 16 January 2018 Accepted: 13 April 2018

Published online: 23 April 2018

\section{References}

1. Becker J, Zelder O, Hafner S, Schroder H, Wittmann C. From zero to hero-design-based systems metabolic engineering of Corynebacterium glutamicum for L-lysine production. Metab Eng. 2011:13:159-68.

2. Park SH, Kim HU, Kim TY, Park JS, Kim SS, Lee SY. Metabolic engineering of Corynebacterium glutamicum for L-arginine production. Nat Commun. 2014;5:4618.

3. Eberhardt D, Jensen JV, Wendisch VF. L-Citrulline production by metabolically engineered Corynebacterium glutamicum from glucose and alternative carbon sources. AMB Express. 2014;4:85.

4. Hirasawa T, Shimizu H. Recent advances in amino acid production by microbial cells. Curr Opin Biotechnol. 2016:42:133-46.

5. Lee JY, Na YA, Kim E, Lee HS, Kim P. The actinobacterium Corynebacterium glutamicum, an industrial workhorse. J Microbiol Biotechnol. 2016:26:807-22.

6. Becker J, Giesselmann G, Hoffmann SL, Wittmann C. Corynebacterium glutamicum for sustainable bioproduction: from metabolic physiology to systems metabolic engineering. Adv Biochem Eng Biotechnol. 2018:162:217-63.

7. Schafer A, Tauch A, Jager W, Kalinowski J, Thierbach G, Puhler A. Small mobilizable multi-purpose cloning vectors derived from the Escherichia coli plasmids pK18 and pK19: selection of defined deletions in the chromosome of Corynebacterium glutamicum. Gene. 1994;145:69-73.

8. Ma W, Wang X, Mao Y, Wang Z, Chen T, Zhao X. Development of a markerless gene replacement system in Corynebacterium glutamicum using upp as a counter-selection marker. Biotechnol Lett. 2015;37:609-17.

9. Wu J, Deng A, Sun Q, Bai H, Sun Z, Shang X, Zhang Y, Liu Q, Liang Y, Liu S, et al. Bacterial genome editing via a designed toxin-antitoxin cassette. ACS Synth Biol. 2018;7:822-31.

10. Shen J, Chen J, Jensen PR, Solem C. A novel genetic tool for metabolic optimization of Corynebacterium glutamicum: efficient and repetitive chromosomal integration of synthetic promoter-driven expression libraries. Appl Microbiol Biotechnol. 2017;101:4737-46.

11. Suzuki N, Nonaka H, Tsuge Y, Inui M, Yukawa H. New multiple-deletion method for the Corynebacterium g/utamicum genome, using a mutant lox sequence. Appl Environ Microbiol. 2005;71:8472-80.

12. Huang $Y, L i L$, Xie S, Zhao N, Han S, Lin Y, Zheng S. Recombineering using RecET in Corynebacterium glutamicum ATCC14067 via a self-excisable cassette. Sci Rep. 2017:7:7916.

13. Jiang W, Bikard D, Cox D, Zhang F, Marraffini LA. RNA-guided editing of bacterial genomes using CRISPR-Cas systems. Nat Biotechnol. 2013;31:233-9. 
14. DiCarlo JE, Norville JE, Mali P, Rios X, Aach J, Church GM. Genome engineering in Saccharomyces cerevisiae using CRISPR-Cas systems. Nucleic Acids Res. 2013:41:4336-43.

15. Oh J-H, van Pijkeren J-P. CRISPR-Cas9-assisted recombineering in Lactobacillus reuteri. Nucleic Acids Res. 2014;42:e131.

16. Cobb RE, Wang Y, Zhao H. High-efficiency multiplex genome editing of Streptomyces species using an engineered CRISPR/Cas system. ACS Synth Biol. 2015;4:723-8

17. Xu T, Li Y, Shi Z, Hemme CL, LiY, Zhu Y, Van Nostrand JD, He Z, Zhou J. Efficient genome editing in Clostridium cellulolyticum via CRISPR-Cas9 nickase. Appl Environ Microbiol. 2015:81:4423-31.

18. Jiang Y, Chen B, Duan C, Sun B, Yang J, Yang S. Multigene editing in the Escherichia coli genome via the CRISPR-Cas9 system. Appl Environ Microbiol. 2015;81:2506-14

19. Li Y, Lin Z, Huang C, Zhang Y, Wang Z, Tang YJ, Chen T, Zhao X. Metabolic engineering of Escherichia coli using CRISPR-Cas9 meditated genome editing. Metab Eng. 2015;31:13-21.

20. Pyne ME, Moo-Young M, Chung DA, Chou CP. Coupling the CRISPR/Cas9 System with lambda Red recombineering enables simplified chromosomal gene replacement in Escherichia coli. Appl Environ Microbiol. 2015;81:5103-14

21. Altenbuchner J. Editing of the Bacillus subtilis genome by the CRISPRCas9 system. Appl Environ Microbiol. 2016;82:5421-7.

22. Westbrook AW, Moo-Young M, Chou CP. Development of a CRISPR-Cas9 tool kit for comprehensive engineering of Bacillus subtilis. Appl Environ Microbiol. 2016;82:4876-95.

23. Nagaraju S, Davies NK, Walker DJ, Kopke M, Simpson SD. Genome editing of Clostridium autoethanogenum using CRISPR/Cas9. Biotechnol Biofuels. 2016:9:219.

24. Wang Y, Zhang ZT, Seo SO, Choi K, Lu T, Jin YS, Blaschek HP. Markerless chromosomal gene deletion in Clostridium beijerinckii using CRISPR/Cas9 system. J Biotechnol. 2015;200:1-5.

25. Jiang Y, Qian F, Yang J, Liu Y, Dong F, Xu C, Sun B, Chen B, Xu X, Li Y, et al. CRISPR-Cpf1 assisted genome editing of Corynebacterium glutamicum. Nat Commun. 2017:8:15179.

26. Cho JS, Choi KR, Prabowo CPS, Shin JH, Yang D, Jang J, Lee SY. CRISPR/ Cas9-coupled recombineering for metabolic engineering of Corynebacterium glutamicum. Metab Eng. 2017;42:157-67.

27. Liu J, Wang Y, Lu Y, Zheng P, Sun J, Ma Y. Development of a CRISPR/Cas9 genome editing toolbox for Corynebacterium glutamicum. Microb Cell Fact. 2017; 16:205

28. Peng F, Wang X, Sun Y, Dong G, Yang Y, Liu X, Bai Z. Efficient gene editing in Corynebacterium glutamicum using the CRISPR/Cas9 system. Microb Cell Fact. 2017:16:201.

29. Min W, Li H, Li H, Liu C, Liu J. Characterization of aspartate kinase from Corynebacterium pekinense and the critical site of arg169. Int J Mol Sci. 2015;16:28270-84.

30. Baumgart M, Unthan S, Ruckert C, Sivalingam J, Grunberger A, Kalinowski J, Bott M, Noack S, Frunzke J. Construction of a prophage-free variant of Corynebacterium glutamicum ATCC 13032 for use as a platform strain for basic research and industrial biotechnology. Appl Environ Microbiol. 2013;79:6006-15

31. Liu X, Homma A, Sayadi J, Yang S, Ohashi J, Takumi T. Sequence features associated with the cleavage efficiency of CRISPR/Cas9 system. Sci Rep. 2016;6:19675

32. Chari R, Yeo NC, Chavez A, Church GM. sgRNA Scorer 2.0: a speciesindependent model to predict CRISPR/Cas9 activity. ACS Synth Biol. 2017;6:902-4

33. Chari R, Mali P, Moosburner M, Church GM. Unraveling CRISPR-Cas9 genome engineering parameters via a library-on-library approach. Nat Methods. 2015;12:823-6.

34. Niimi S, Suzuki N, Inui M, Yukawa H. Metabolic engineering of 1,2-propanediol pathways in Corynebacterium glutamicum. Appl Microbiol Biotechnol. 2011:90:1721-9.

35. Siebert D, Wendisch VF. Metabolic pathway engineering for production of 1,2-propanediol and 1-propanol by Corynebacterium glutamicum. Biotechnol Biofuels. 2015;8:91.
36. Zhang Y, Cai J, Shang X, Wang B, Liu S, Chai X, Tan T, Zhang Y, Wen T. A new genome-scale metabolic model of Corynebacterium glutamicum and its application. Biotechnol Biofuels. 2017;10:169.

37. Reddy GK, Wendisch VF. Characterization of 3-phosphoglycerate kinase from Corynebacterium glutamicum and its impact on amino acid production. BMC Microbiol. 2014;14:54

38. Shang X, Chai X, Lu X, Li Y, Zhang Y, Wang G, Zhang C, Liu S, Zhang Y, Ma J, Wen T. Native promoters of Corynebacterium glutamicum and its application in L-lysine production. Biotechnol Lett. 2018:40:383-91.

39. Wang $H$, Yang $H$, Shivalila CS, Dawlaty MM, Cheng AW, Zhang F, Jaenisch R. One-step generation of mice carrying mutations in multiple genes by CRISPR/Cas-mediated genome engineering. Cell. 2013;153:910-8.

40. Cong L, Ran FA, Cox D, Lin S, Barretto R, Habib N, Hsu PD, Wu X, Jiang W, Marraffini LA, Zhang F. Multiplex genome engineering using CRISPR/Cas systems. Science. 2013;339:819-23.

41. Yang P, Wang J, Qi Q. Prophage recombinases-mediated genome engineering in Lactobacillus plantarum. Microb Cell Fact. 2015;14:154.

42. Binder S, Siedler S, Marienhagen J, Bott M, Eggeling L. Recombineering in Corynebacterium glutamicum combined with optical nanosensors: a general strategy for fast producer strain generation. Nucleic Acids Res. 2013;41:6360-9.

43. Kusano K, Takahashi NK, Yoshikura H, Kobayashi I. Involvement of RecE exonuclease and RecT annealing protein in DNA double-strand break repair by homologous recombination. Gene. 1994;138:17-25.

44. Bassalo MC, Garst AD, Halweg-Edwards AL, Grau WC, Domaille DW, Mutalik VK, Arkin AP, Gill RT. Rapid and efficient one-step metabolic pathway integration in E. coli. ACS Synth Biol. 2016;5:561-8.

45. Zhang G, Wang W, Deng A, Sun Z, Zhang Y, Liang Y, Che Y, Wen T. A mimicking-of-DNA-methylation-patterns pipeline for overcoming the restriction barrier of bacteria. PLoS Genet. 2012;8:e1002987.

46. Nakamura J, Kanno S, Kimura E, Matsui K, Nakamatsu T, Wachi M. Temperature-sensitive cloning vector for Corynebacterium glutamicum. Plasmid. 2006:56:179-86.

47. Sambrook J, Russell D. Molecular cloning: a laboratory manual. Cold Spring Harbor: Cold Spring Harbor Laboratory Press; 2001.

48. Gibson DG, Young L, Chuang RY, Venter JC, Hutchison CA 3rd, Smith HO Enzymatic assembly of DNA molecules up to several hundred kilobases. Nat Methods. 2009:6:343-5.

49. Liu H, Ye R, Wang YY. Highly efficient one-step PCR-based mutagenesis technique for large plasmids using high-fidelity DNA polymerase. Genet Mol Res. 2015;14:3466-73.

50. Tauch A, Kirchner O, Loffler B, Gotker S, Puhler A, Kalinowski J. Efficient electrotransformation of corynebacterium diphtheriae with a mini-replicon derived from the Corynebacterium glutamicum plasmid pGA1. Curr Microbiol. 2002;45:362-7.

51. Shang $X$, Zhang $Y$, Zhang G, Chai X, Deng A, Liang Y, Wen T. Characterization and molecular mechanism of AroP as an aromatic amino acid and histidine transporter in Corynebacterium glutamicum. J Bacteriol. 2013:195:5334-42.

52. Menkel E, Thierbach G, Eggeling L, Sahm H. Influence of increased aspartate availability on lysine formation by a recombinant strain of Corynebacterium glutamicum and utilization of fumarate. Appl Environ Microbiol. 1989:55:684-8.

Ready to submit your research? Choose BMC and benefit from

- fast, convenient online submission

- thorough peer review by experienced researchers in your field

- rapid publication on acceptance

- support for research data, including large and complex data types

- gold Open Access which fosters wider collaboration and increased citations

- maximum visibility for your research: over 100M website views per year

At BMC, research is always in progress.

Learn more biomedcentral.com/submissions 